\title{
IMPROVEMENTS, EVALUATION, AND APPLICATION OF 1D VETEM INVERSION AND DEVELOPMENT AND APPLICATION OF 3D VETEM INVERSION TO WASTE PITS AT THE IDAHO NATIONAL ENGINEERING AND ENVIRONMENTAL LABORATORY
}

\author{
FINAL REPORT \\ for Period August 1, 2001 to July 31, 2004
}

\author{
WENG CHO CHEW \\ Center for Computational Electromagnetics \\ and Electromagnetics Laboratory \\ Department of Electrical and Computer Engineering \\ University of Illinois at Urbana-Champaign \\ Urbana, IL 61801-2991 \\ Tel: (217) 333-7309 \\ Fax: (217) 244-7345 \\ E-mail: w-chew@uiuc.edu
}

October 27, 2004

Prepared for

THE U.S. DEPARTMENT OF ENERGY

AGREEMENT NO. DE-FG07-01ID14132 


\section{PROJECT INFORMATION}

AWARD NUMBER: DE-FG07-01ID14132

AWARDEE NAME: University of Illinois, Urbana-Champaign

PROJECT OR AWARDEE ACCOUNT NO.: Acct. 1-0-28690

PROJECT TITLE: Improvements, Evaluation, and Applications of 1D VETEM Inversion and Development and Application of 3D VETEM Inversion to Waste Pits at the Idaho National Engineering and Environmental Laboratory

PRINCIPLE INVESTIGATOR: Dr. Weng Cho Chew

REPORT CATEGORY: Scientific/Technical

REPORT NAME: Final Scientific/Technical Report

FREQUENCY: Final

PERIOD COVERED: August 12001 - July 31, 2004

DUE DATE: October 29, 2004 


\section{DISCLAIMER}

This report was prepared as an account of work sponsored by an agency of the United States Government. Neither the United States nor the Department of Energy, nor any agency thereof, or any of their employees, makes any warranty, expressed or implied, or assumes any legal liability or responsibility for the accuracy, completeness, or usefulness of any information, apparatus, product or process disclosed or represents that its use would not infringe privately-owned rights. Reference herein to any specific commercial product, process, or service by trade name, trademark, manufacturer, or otherwise, does not necessarily constitute or imply its endorsement, recommendation, or favoring by the United States Government or any agency thereof. The views and opinions of authors expressed herein do not necessarily state or reflect those of the United States Government or any agency thereof.

Portions of this document may be illegible in electronic image products. Images are produced from the best available original document. 


\section{Contents}

PROJECT INFORMATION I

$\begin{array}{ll}\text { DISCLAIMER } & \text { II }\end{array}$

LIST OF CONTENTS III

1 EXECUTIVE SUMMARY 1

2 RESEARCH OBJECTIVES 2

3 METHODS AND RESULTS 3

3.1 VETEM System and Data Preprocessing . . . . . . . . . 5

3.1.1 System Function of the VETEM System . . . . . . . 5

3.1.2 Background Estimation . . . . . . . . . . . . . 8

3.1.3 Raw Waveform Calibration ............ 9

3.2 1D Inversion with the Distorted Born Iterative Method . . . . 13

3.2.1 Simulation of the 1D Solver in a 2D Environment . . . 19

3.2.2 Simulation of the 1D Solver in a 3D Environment . . . 19

3.2.3 Application of 1D Inversion to Field Data . . . . . . . 20

3.2.4 Computational Complexity . . . . . . . . . . . . 21

3.3 3D Inversion by the Born Approximation and the Distorted

Born Iterative Method . . . . . . . . . . . . . . . 30

3.3.1 Half-space Volume Integral Equation . . . . . . . . . . 30

3.3.2 3D Half-space Distorted Born Iterative Method . . . . 32

3.3.3 Localized 3D Inversion . . . . . . . . . . . . . . 36

3.3.4 Validation of 3D Inversion with Synthetic Data . . . 37

3.3.5 Application of 3D Inversion to Field Data . . . . . . 45

3.4 Conclusions . . . . . . . . . . . . . . . . 51 
4 PUBLICATIONS

ACKNOWLEDGEMENTS 57

BIBLIOGRAPHY 58 


\section{Chapter 1}

\section{EXECUTIVE SUMMARY}

The project aim was the improvement, evaluation, and application of one dimensional (1D) inversion and development and application of three dimensional (3D) inversion to processing of data collected at waste pits at the Idaho National Engineering and Environmental laboratory. The inversion methods were intended mainly for the Very Early Time Electromagnetic (VETEM) system which was designed to improve the state-of-the-art of electromagnetic imaging of the shallow (0 to about $5 \mathrm{~m}$ ) subsurface through electrically conductive soils. They are also capable of processing data from other low frequency systems, such as GEM-2 or GEM-3. The proposed objectives have been achieved by developing new numerical modeling to more fully exploit and interpret field data. We have not only processed VETEM data gathered at Pit 9, but also have processed GEM-2 data gathered at Pit 10. The numerical modelings performed include 1D and 3D model appropriate to the low frequency electromagnetic system. A 1D inversion with the Distorted Born Iterative Method (DBIM) has been developed and improved. A 3D inversion based on the Born approximation was developed, followed by a 3D inversion baed on DBIM. These methods offer significant 1D and 3D electromagnetic imaging capabilities in the shallow subsurface, and now are available for use at DOE sites that have shallow subsurface imaging needs. Products of this research are documented in many papers, applicable to electromagnetic subsurface imaging, and suggest further research and development. 


\section{Chapter 2}

\section{RESEARCH OBJECTIVES}

Data processing is crucial to reconstruction of the subsurface imaging and understanding the subsurface situation. Many DOE facilities are in need of subsurface imaging in conductive zones. For example, the characterization of waste pits, mapping the complex geological/hydrological framework of the conductive zones, and the detection and characterization of metal and concrete structures. 1D inversion has been used as a matured technique for data processing for a long time. The advantage of $1 \mathrm{D}$ model is its high efficiency, and therefore it is suited for real-time processing and helpful to general evaluation of the subsurface situation. 3D model accounts for more physics of the interaction of the electromagnetic waves with the buried objects and hence is able to give more reliable information about the subsurface situation. The disadvantage of the 3D model is that it is often time-consuming, especially when rigorous model is used. The tradeoff may be the one that uses a simple model, such as Born approximation. Our first objective is therefore the development of a 3D inversion method based on the Born approximation. Also we are intended to develop a more rigorous 3D inversion method based on DBIM. The application of DBIM leads to drastically large computational overhead. The efficient forward modelling and localized inversion are proposed to help cut down on expenditure. Besides the efficiency, people are also concerned about the stability of the inversion method for practical application, which is addressed by choosing the stabilizer appropriate to the low frequency electromagnetic inversion. 


\section{Chapter 3}

\section{METHODS AND RESULTS}

Subsurface imaging has been of paramount interest in near-surface geophysical explorations, environmental explorations, and detection of unexploded ordinance. The associated imaging problem is quite challenging due to the considerable loss in the earth and the limited-angle nature of data acquisition. Most subsurface imaging systems fall into two broad categories: ground penetrating radar (GPR) and electromagnetic induction (EMI) systems. Higher operation frequencies suggest that GPR will have considerably better resolution, yet GPR cannot penetrate lossy earth very well. On the other hand, EMI sensors operate in the kilohertz frequencies, which yield a very poor resolution. A very early time electromagnetic (VETEM) system is a compromise developed by the U.S. Geological Survey (USGS) that operates in a "middle" frequency regime. This is a time domain system that employs a ramp input, the energy of which is concentrated below $5 \mathrm{MHz}$.

In practice, a time domain, single transmitter-receiver pair traversing a region of interest in unison is much more convenient and feasible to operate than a multiple receiver system. Although the work here can be applied to the latter case, we will mainly consider the former configuration.

The methods detailed in this chapter assume time-harmonic, frequency domain input. Hence, in the case of VETEM, we actually process the Fouriertransform of measurement data, rather than the time domain waveforms themselves.

In Section 3.1, we discuss the very early time electromagnetic (VETEM) system and its calibration [1]. Then we present an experimental and a numerical method to estimate the soil conductivity in Section 3.1.2. In Section 3.1.3, we detail the waveform processing before the actual inversion, which is 
indispensable because of practical problems concerning noise, slight configuration mismatches, inhomogeneous background, and the mostly inductive transfer function of the data acquisition system. In Section 3.2, we give the 1D inverse solver for vertical magnetic dipole (VMD) and horizontal magnetic dipole (HMD) contributions at the receiver. In Sections 3.2.1 and 3.2.2, we demonstrate the viability of $1 \mathrm{D}$ multifrequency inversion in $2 \mathrm{D}$ and $3 \mathrm{D}$ environments, respectively, by employing 2D and 3D CGFFT forward solvers to obtain synthetic data. The 1D inversion is then applied to VETEM data obtained through measurements over a region of $40 \mathrm{~m}$ by $90 \mathrm{~m}$ and compare our inversion with a shaded relief late-time image, which, we believe, is in agreement with our inversion. We comment on the computational complexity in Section 3.2.4.

In Section 3.3, we discuss how preprocessing is performed over subdomains of the region of interest for a 3D inversion even though inverting the whole domain by a $3 \mathrm{D}$ method would be prohibitively intensive. Formulation and results of the rigorous 3D DBIM method are presented. Finally, some conclusions are drawn in Section 3.4. 


\subsection{VETEM System and Data Preprocessing}

The major motivation for the VETEM system is to penetrate into conductive earth deeper than the ground penetrating radar while obtaining better resolution in the first $5 \mathrm{~m}$ than the existing electromagnetic induction systems. For this purpose, a ramp input, shown in Figure 3.1, is employed with most of the spectral energy below $5 \mathrm{MHz}$. Also, the receiver is kept perpendicular to avoid the direct coupling. The VETEM system is depicted in Figure 3.2. Accurate 3D numerical simulations of similar enhanced systems can be found in $[2]$.
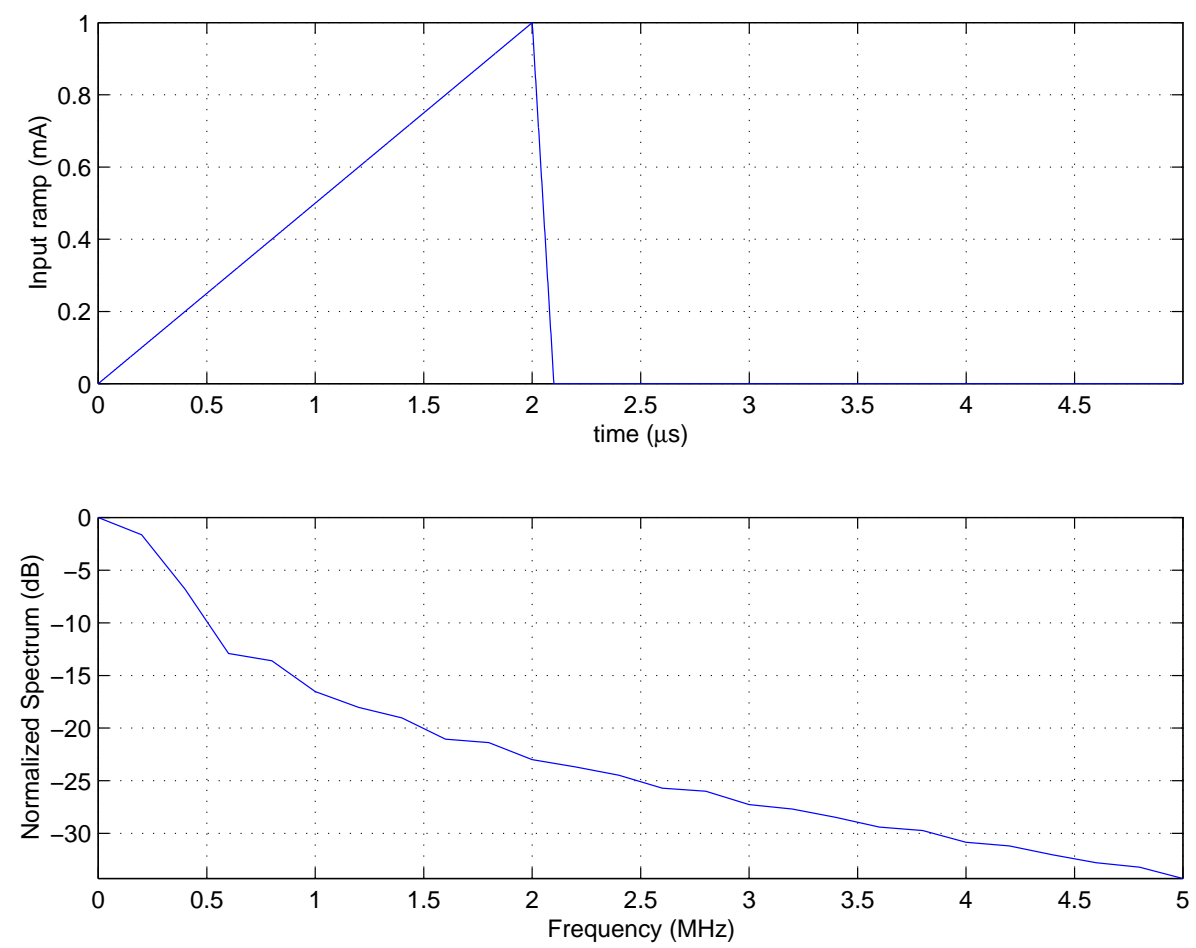

Figure 3.1: The input ramp of VETEM and its spectrum.

\subsubsection{System Function of the VETEM System}

It is assumed that the VETEM system, consisting of two square loops, can be lumped into two magnetic dipoles, which is expedient for numerical mod- 


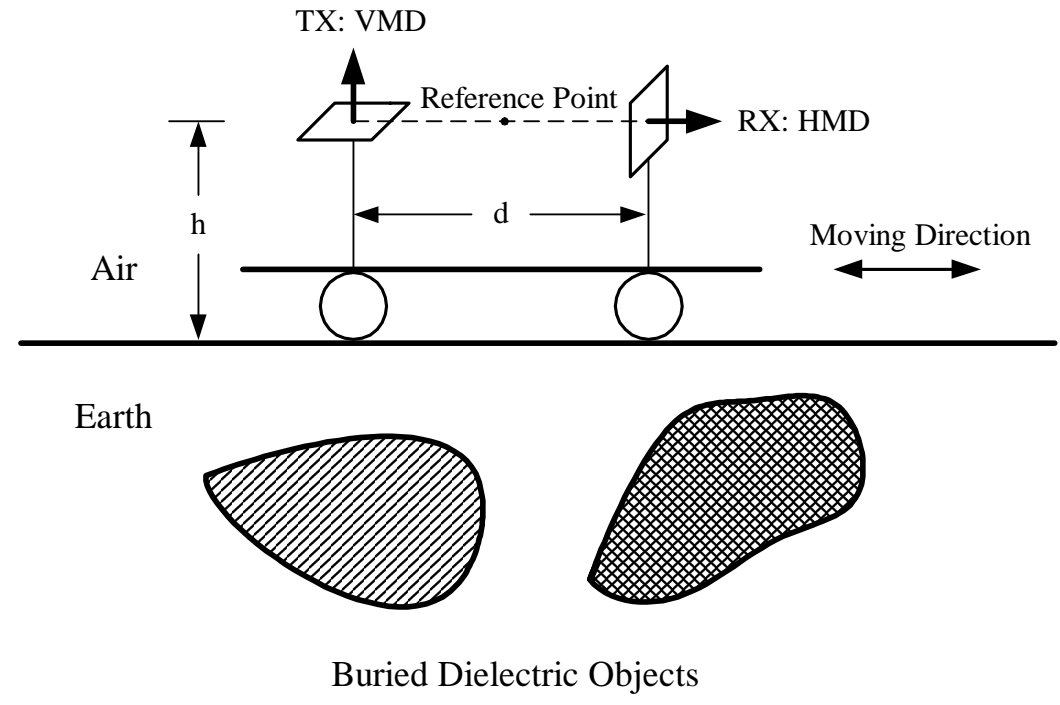

Figure 3.2: The schematic diagram of the VETEM system with $d=2 \mathrm{~m}$.

elling. However, it is equivalent to saying that the system function is a delta function, which is evidently wrong. In order to be able to process the data in 1D, all 3D effects need to be approximately accounted for by a deconvolution of the system function $[3,4]$. This deconvolution is performed pointwise, and provided that the computationally less intensive calibration is started before the 1D inversion, calibration and inversion can be performed simultaneously. Assuming that the system circuitry is linear, one can lump its effects such that the transmitter and receiver antennas can be considered to be two magnetic dipoles. The VETEM system of USGS radiates most of its energy at frequencies under $5 \mathrm{MHz}$ [1], thus facilitating the dipole approximation. In order to determine the system function, the VETEM system has been run over a metal plate, in which case the resultant field can be expressed analytically. If we let $L(\omega)=S(\omega) H(\omega) R(\omega)$ be the measured response, in which $H(\omega)$ is the analytical impulse response, and $R(\omega)$ is the input current ramp, we can obtain the system function as $S(\omega)=\frac{L(\omega)}{H(\omega) R(\omega)}$. This system function is then deconvolved from each measured waveform to rid the waveform of spurious effects.

In the case of a ground plane, the response of the image can be analytically expressed as

$$
H(k r)=-\frac{I A}{4 \pi} \frac{e^{i k r}}{r^{5}} \rho\left|z+z^{\prime}+2 d_{1}\right| f(k r),
$$


where $f(x)=x^{2}+3 i x-3$ and $r=\sqrt{\left(z+z^{\prime}+2 d_{1}\right)^{2}+\rho^{2}}$ [5]. We note that this system function will lump together effects due to circuitry and the mostly inductive transfer function between the 3D loops, and hence will not be identical to a 3D MoM simulation of the VETEM system.

(a)

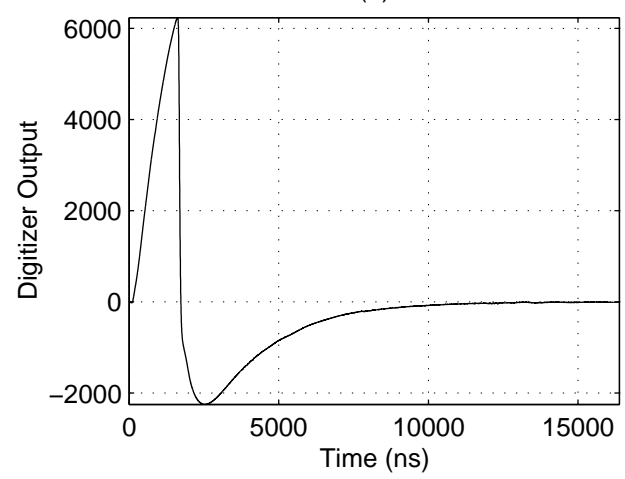

(c)

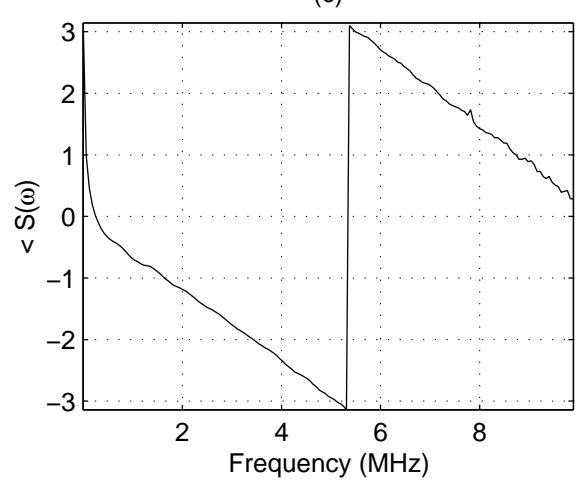

(b)

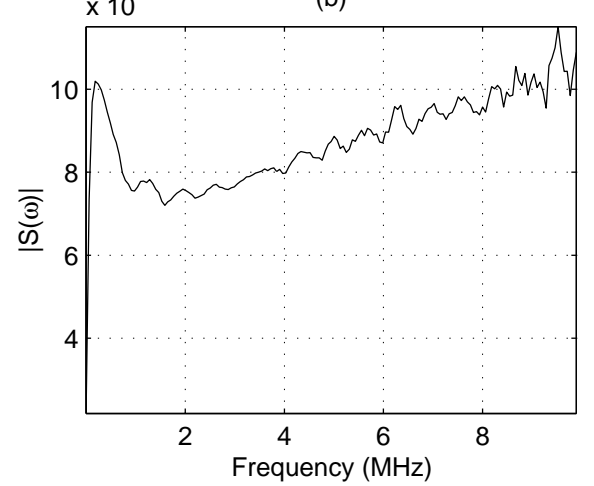

(d)

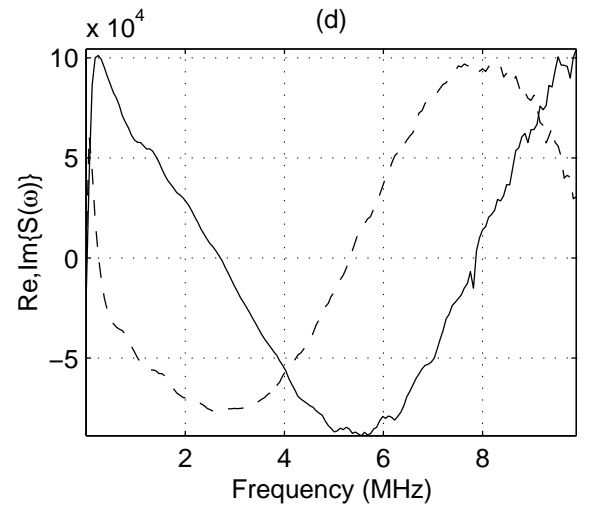

Figure 3.3: Calibration of the VETEM system. (a) Calibration waveform. (b) Amplitude of the system function. (c) Phase of the system function. (d) Real (solid) and imaginary (dashed) parts of the system function.

The VETEM system has been run over a metal plate to obtain the calibration waveform in Figure 3.3(a). This waveform reflects the physics when the receiver loop is almost perfectly perpendicular or the tilt angle $\theta$ is slightly nonzero. As soon as the current is turned off, induced currents start to circulate in the opposite direction, which leads to the tail in the calibration waveform. Yet, most other data from the VETEM system looks considerably different. The tail due to Lenz's effect is observed right after a negative-going 
ramp. Indeed, the negative-going ramp is due to a strong VMD primary contribution, which is due to the tilt of the receiver coil.

According to our current calibration in Figure 3.3(b-d), the system function manifests some capacitive effects, apart from the expected inductive coupling.

\subsubsection{Background Estimation}

One way to estimate the background at each measurement point is to use the initial slope of VETEM waveforms [6]. We have found that the slope is an increasing function of background conductivity. This can be readily seen for the limiting cases of perfect conductor and perfect insulator. The former will have exactly the same slope as the input ramp due to the image source, whereas the latter will not induce any currents. In Figure 3.4, we plotted several waveforms under different background conductivities. We note that this estimation yields an average value for soil conductivity. That is, in the presence of highly conductive scatterers, the soil conductivity will be somewhat overestimated.

Although this method is quite simple, it requires a precise calibration. Instead, we propose the following method, which retains the computational simplicity of the preprocessing stage [7].

1. Choose the highest possible frequency for which the dipole approximation is valid, yet the skin depth $\delta$ is as small as possible.

2. Since the skin depth is small, the subsurface scattered magnetic field $H^{s}$ can be ignored. Then we can write the total magnetic field in terms of the estimated tilt angle $\theta$ as

$$
-H_{z}^{P} \sin (\theta)+H_{x}^{R}\left(\sigma_{b}\right) \cos (\theta)-H_{z}^{R}\left(\sigma_{b}\right) \sin (\theta)=f\left(\sigma_{b}\right) \approx H^{\text {meas }},
$$

in which $H_{z}^{P}$ has an analytic expression, and $H_{x}^{R}, H_{z}^{R}$ are expressed by the Sommerfeld integrals (3.2) and (3.3), respectively.

3. Solve the equation $f\left(\sigma_{b}\right)-H^{\text {meas }}=0$ for the background conductivity $\sigma_{b}$ by using a root solver.

Convergence to the background value is rather fast when the error bound is $0.1 \mathrm{mS} / \mathrm{m}$. Still, we note that the root solver yields an effective background conductivity because we do not employ frequencies larger than $5 \mathrm{MHz}$ in order to retain the dipole approximation. 


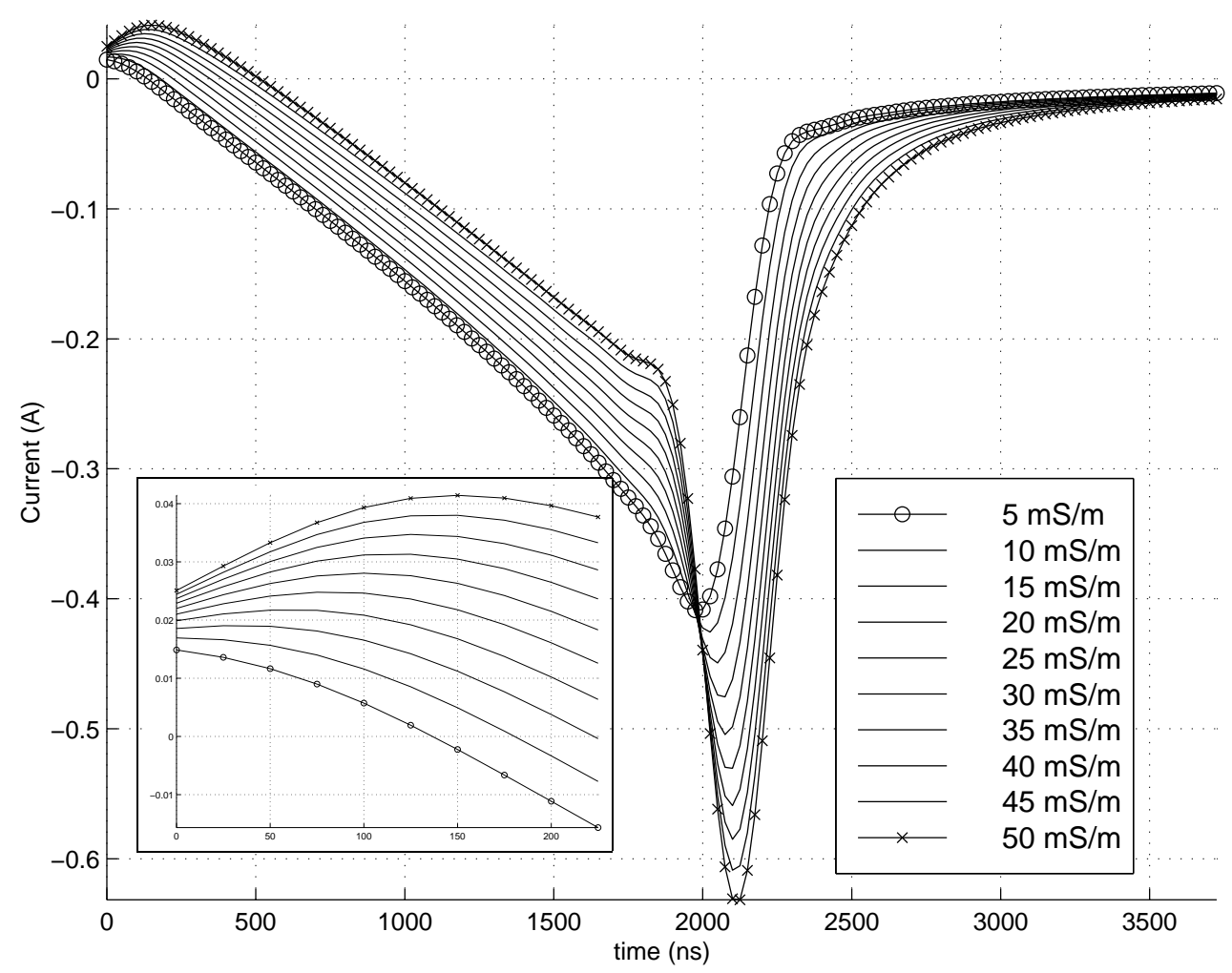

Figure 3.4: Synthetic waveforms obtained by a 3D MoM model of the VETEM system for a set of background values, $\sigma_{b}=5-50 \mathrm{mS} / \mathrm{m}, \theta=4^{\circ}$. A $1 \mathrm{~m} \times 1$ $\mathrm{m}, 1-\mathrm{m}$-deep scatterer is assumed. Background estimation could rely on the very early time response, which is shown on the lower-left figure.

\subsubsection{Raw Waveform Calibration}

After the background conductivity is estimated, the VETEM system function is deconvolved from the measured data. Because the receiving antenna is never perfectly perpendicular, it is necessary to extract VMD primary field as well as VMD and HMD half-space reflections from the measured data. In the following expressions where the superscripts "R" and "I" denote reflected and image fields, respectively, we extract the images from half-space reflections by letting $R_{12}=T_{12}-1$ and derive closed-forms for them by using the Sommerfeld identity. 


$$
\begin{gathered}
H_{x}^{R}=H_{x}^{I}+H_{x}^{R^{\prime}}=-\frac{I A}{4 \pi} \frac{e^{i k_{1} r}}{r^{5}} \rho\left|z+z^{\prime}+2 d_{1}\right| f\left(k_{1} r\right)- \\
\frac{I A}{4 \pi} \int_{0}^{\infty} d k_{\rho} k_{\rho}^{2} J_{1}\left(k_{\rho} \rho\right) T_{12} e^{i k_{1 z}\left(z+z^{\prime}+2 d_{1}\right)} \\
H_{z}^{R}=H_{z}^{I}+H_{z}^{R^{\prime}}=-\frac{I A}{4 \pi} \frac{e^{i k_{1} r}}{r^{5}}\left\{2 r^{2}\left(i k_{1} r-1\right)-\rho^{2} f\left(k_{1} r\right)\right\}- \\
\frac{i I A}{4 \pi} \int_{0}^{\infty} d k_{\rho} \frac{k_{\rho}^{3}}{k_{1 z}} J_{o}\left(k_{\rho} \rho\right) T_{12} e^{i k_{1 z}\left(z+z^{\prime}+2 d_{1}\right)},
\end{gathered}
$$

where $r=\sqrt{\left(z+z^{\prime}+2 d_{1}\right)^{2}+\rho^{2}}$ and $f(x)=x^{2}+3 i x-3[5]$.

Hence, the factor $H_{x}^{R} \cos (\theta)-\left(H_{z}^{R}+H_{z}^{P}\right) \sin (\theta)$ is subtracted from the deconvolved waveform where the VMD primary contribution $H_{z}^{P}$ is equal to $-H_{z}^{I}$ with $r=\sqrt{\left(z-z^{\prime}\right)^{2}+\rho^{2}}$.

An antenna configuration that keeps the receiver perpendicular to the transmitter is desirable to avoid direct coupling. However, even slight tilts can result in considerable direct coupling. It is therefore important to estimate these tilts and undo the primary coupling. This is achieved by noting that unless the scatterers are perfectly conducting, the quasi-magnetostatic contribution to the waveform is due to the direct coupling. The tilt angle can be computed by noting that the field will be predominantly a magnetostatic primary field for very low frequencies. Hence, by extrapolating the scattering data to obtain the magnetostatic field $H_{o}$, the angle can be estimated. For low frequencies, $H_{z}^{P} \approx \frac{I A}{4 \pi \rho^{3}}$. Therefore,

$$
\theta \approx-\sin ^{-1}\left(\frac{4 \pi \rho^{3} H_{o}}{I A}\right) .
$$

This is readily seen by noting that the TE Fresnel reflection tends to zero as the frequency goes to zero; i.e., half-space reflected and scattered fields do not have any DC component. Nevertheless, this effect cannot be readily captured experimentally because the receiver loop has a small, finite resistance. Denoting the induced voltage at the receiver by $V_{r}=j \omega L^{\prime} I_{t}$, the current at the receiver will be

$$
I_{r}=\frac{V_{r}}{j \omega L+R}=\frac{j \omega L^{\prime} I_{t}}{j \omega L+R}
$$


provided that it has an inductance $L$ and resistance $R$. It is readily seen that the expression has a nonzero limit as $\omega$ goes to zero if and only if $R$ is zero. Hence, to estimate the extent of DC coupling, it suffices to employ the lowest frequency for which $\omega L \gg R$. For the VETEM waveforms, this frequency has been in the $60-70 \mathrm{kHz}$ range. Akin to (3.4), it suffices to note the relationship $I_{\text {meas }}=I_{\text {comp }} \sin (\theta)$ to find the tilt angle, in which $I_{\text {comp }}$ is obtained by magnetostatics.

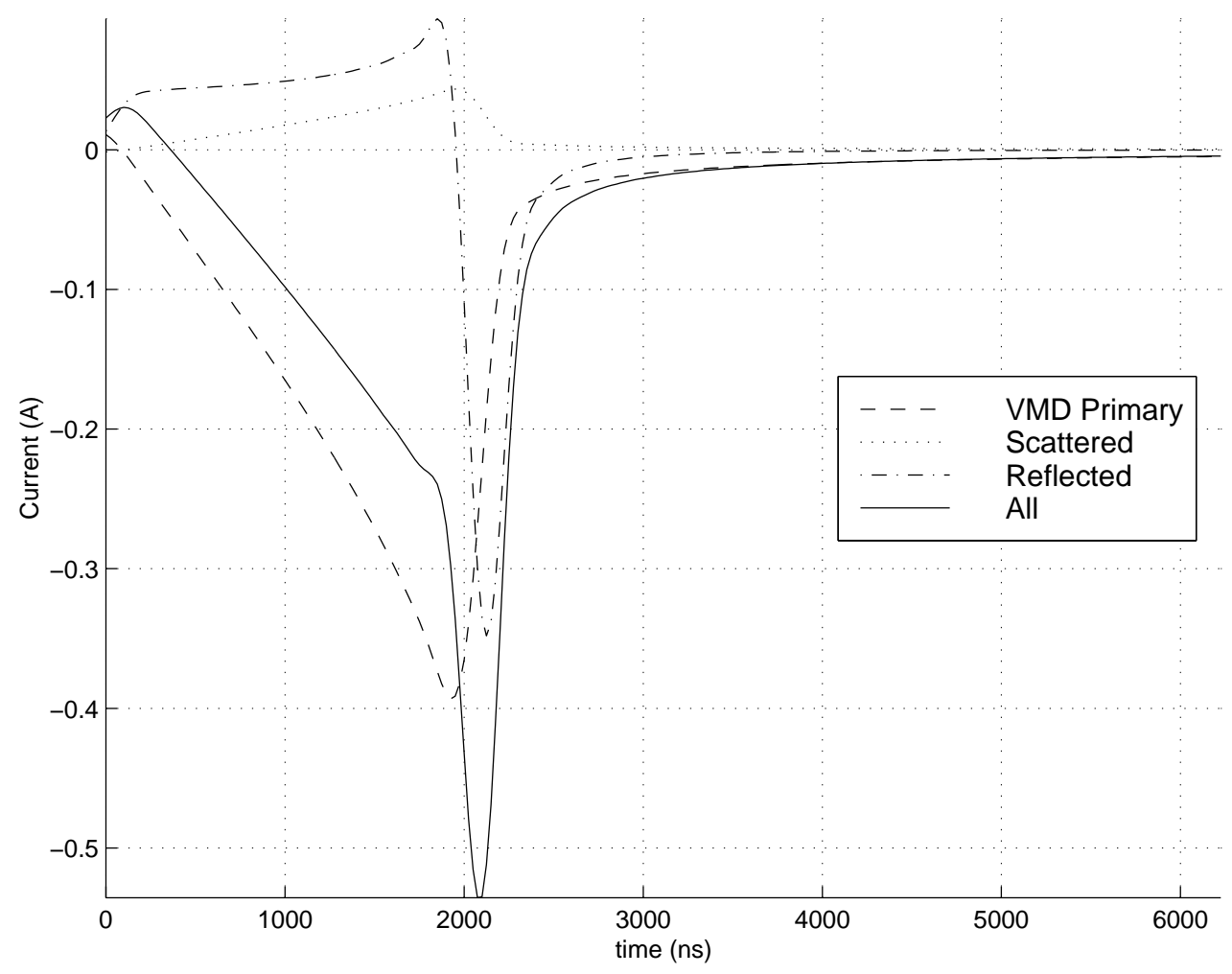

Figure 3.5: Waveforms simulated by a $3 \mathrm{D}$ MoM model compare very well with most VETEM raw waveforms. Here, the receiver angle is $4^{o}$ and $\sigma_{b}=30 \mathrm{mS} / \mathrm{m}$. A $1 \mathrm{~m} \times 1 \mathrm{~m}, 1$-m-deep scatterer is assumed. Constituents of the waveform are also shown.

We show in Figure 3.5 that waveforms identical to VETEM raw waveforms can be synthesized by a 3D MoM solver if the angle is nonzero. These look quite similar to the actual raw waveforms in Figure 3.6(a). In this latter figure, the preprocessing of actual waveforms can be followed in the time 
domain. Figure 3.6(b) shows the waveforms after the deconvolution of the system function. Figure 3.6(c) is the computed VMD primary and HMD, VMD reflected fields convolved with the measured ramp which need to be subtracted from the waveform. The result of this subtraction is shown in Figure 3.6(d). Corresponding reconstructions of the conductivity profile by the $1 \mathrm{D}$ solver are in Figure 3.6(e).

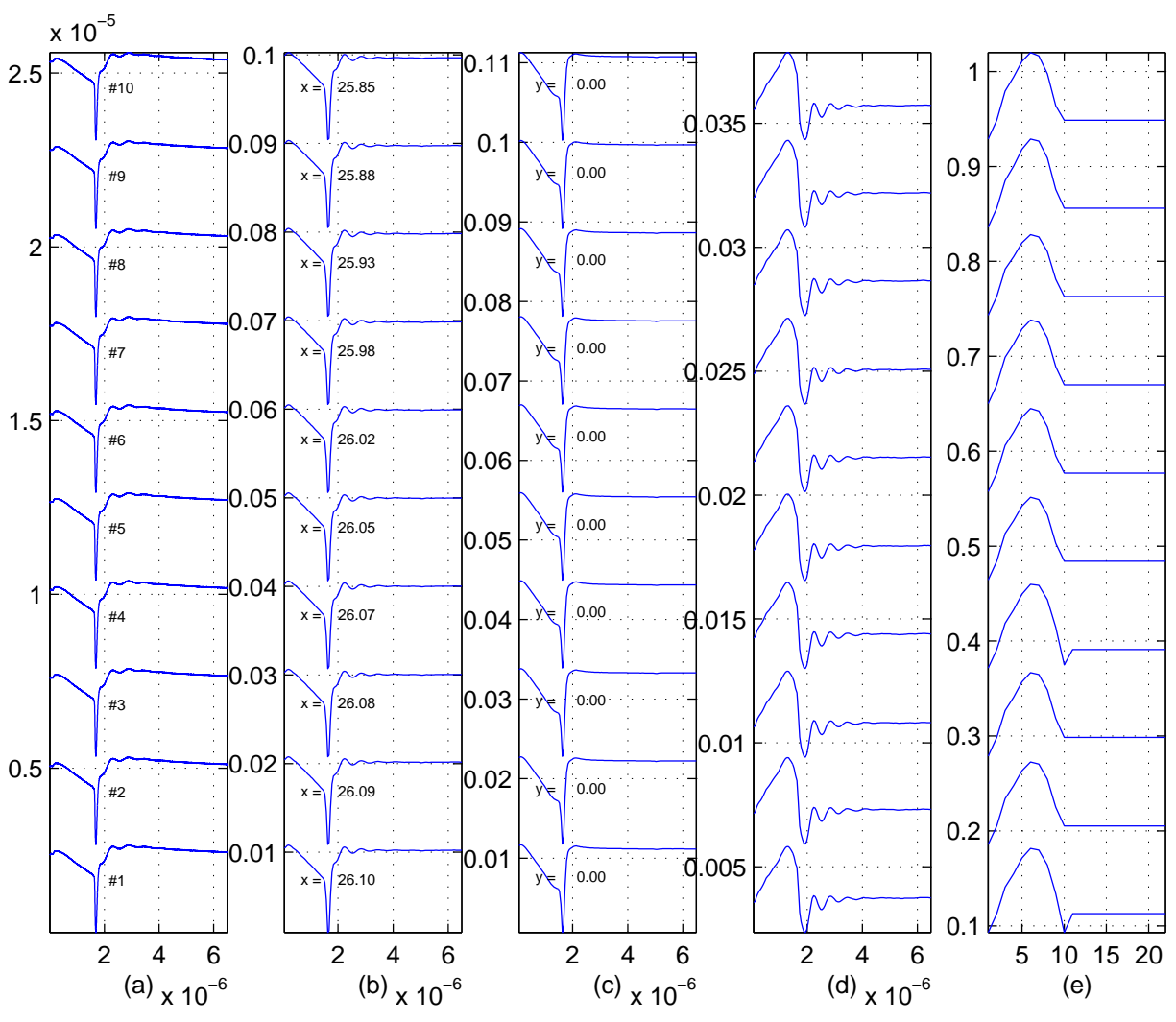

Figure 3.6: Calibration of a sequence of raw waveforms. (a) Subsequent VETEM waveforms along a traversal. (b) After system function deconvolution. (c) Halfspace reflections plus primary field convolved with the ramp input. (d) After the subtraction of half-space reflections and VMD primary. (e) Corresponding reconstructions. 


\subsection{D Inversion with the Distorted Born It- erative Method}

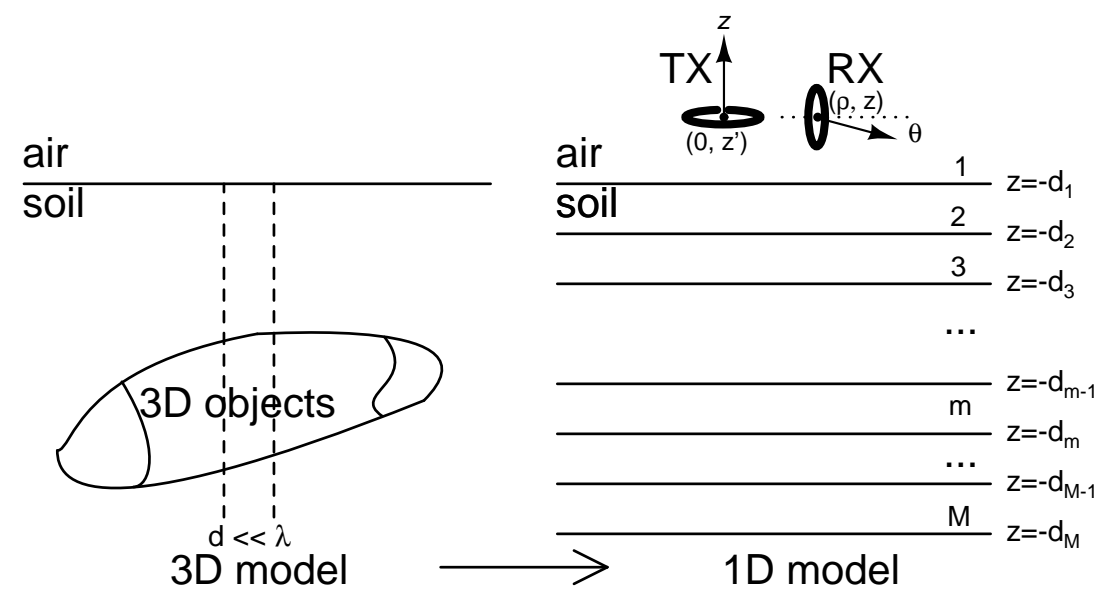

Figure 3.7: The approximation of a local 3D problem by a 1D multilayer problem. The transmitter (TX) and receiver (RX) loops are a VMD and a possibly tilted HMD.

In the subsurface imaging of 3D large-scale buried structure, a small portion of the 3D model can be locally simplified to a $1 \mathrm{D}$ model, as shown in Figure 3.7. Here, a layered geometry is used to approximate the buried target in the relevant small region. The uppermost layer denoted by $m=2$ is fixed to the given soil parameters, and the region $m=1$ corresponds to the air half-space. The transmitter is a horizontal loop (VMD) and the receiver is an almost vertical loop with a tilt angle $\theta$. This is necessary because of the practical problem of keeping the receiver loop perfectly vertical. The multifrequency scattered field data obtained by the actual VETEM system is inverted by using a 1D distorted Born iterative method (DBIM), which utilizes the Born approximation while updating the background parameters $[8-12]$.

The method in $1 \mathrm{D}$ can be illustrated readily as follows. In the spectral domain, the relevant 1D differential equation is

$$
\left[\frac{d^{2}}{d z^{2}}+k_{z}^{2}\right] \tilde{g}_{z}=-c \delta\left(z-z^{\prime}\right)
$$


in which $\tilde{g}_{z}$ is the Green's function and $c$ is a constant that can be easily determined by matching the singularity at $z=z^{\prime}$, i.e., when the source point coincides with the observation point. A similar equation can be written for the background medium:

$$
\left[\frac{d^{2}}{d z^{2}}+k_{z b}^{2}\right] \tilde{g}_{z b}=-c \delta\left(z-z^{\prime}\right)
$$

Here, the background is also inhomogeneous. By subtracting one equation from the other and noting that

$$
k_{z}^{2}=k_{z b}^{2}+\delta k^{2}
$$

we obtain

$$
\left[\frac{d^{2}}{d z^{2}}+k_{z b}^{2}\right]\left(\tilde{g}_{z}-\tilde{g}_{z b}\right)=-\delta k^{2} \tilde{g}_{z}\left(k_{\rho}, z, z^{\prime}\right)
$$

Finally, by invoking the background Green's function on this equivalent current, we arrive at

$$
\Delta \tilde{g}_{z}=\tilde{g}_{z}-\tilde{g}_{z b}=\frac{1}{c} \int_{S} d z^{\prime \prime} \tilde{g}_{z b}\left(k_{\rho}, z, z^{\prime \prime}\right) \tilde{g}_{z}\left(k_{\rho}, z^{\prime \prime}, z^{\prime}\right) \delta k^{2}\left(z^{\prime \prime}\right),
$$

where $S$ is the bounded region in which $\delta k^{2}$ is allowed to be nonzero. The above equation is in fact a nonlinear integral equation since $\tilde{g}_{z}$ is related to $\delta k^{2}$. By using the distorted Born approximation

$$
\tilde{g}_{z}\left(k_{\rho}, z, z^{\prime}\right) \approx \tilde{g}_{z b}\left(k_{\rho}, z, z^{\prime}\right),
$$

the nonlinear equation can be linearized.

Given an initial guess of the inhomogeneous background, the 1D inverse solver corresponding to the problem in Figure 3.7 can be written as

$$
\Delta H=H^{\text {meas }}-H_{x}(\rho, z) \cos (\theta)+H_{z}(\rho, z) \sin (\theta)=\sum_{m=3}^{M} F_{m}\left(\rho, z, z^{\prime}\right) \delta k_{m}^{2}
$$

where $H_{x}$ and $H_{z}$ are respectively the computed scattered HMD and VMD fields, which are free of half-space reflections, and $\delta k_{m}^{2}$ are the unknowns. Here, $M$ is the number of $1 \mathrm{D}$ layers, each $d_{m}$ meters thick, and $F_{m}$ is the Fréchet derivative corresponding to the $m$ th layer. In the following expressions, $k_{m z}=\sqrt{k_{m}^{2}-k_{\rho}^{2}}, k_{m}^{2}=k_{o}^{2} \mu_{m}\left(\epsilon_{m}+\frac{i \sigma_{m}}{\omega \epsilon_{o}}\right)$; and $R_{i j}, T_{i j}, \widetilde{R}_{i j}, \widetilde{T}_{i j}$ denote 
TE Fresnel reflection, transmission, generalized reflection, and generalized transmission coefficients, respectively.

The scattered fields are given by

$$
\begin{aligned}
& H_{x}=\left(-\frac{I A}{4 \pi}\right) \int_{0}^{\infty} d k_{\rho} k_{\rho}^{2} J_{1}\left(k_{\rho} \rho\right) \underbrace{\left[\frac{T_{12} \widetilde{R}_{23} T_{21} e^{i k_{2 z}\left(d_{2}-d_{1}\right)}}{\left.1+R_{12} \widetilde{R}_{23} e^{i k_{2 z}\left(d_{2}-d_{1}\right)}\right]} e^{i k_{1 z}\left(z+z^{\prime}+2 d_{1}\right.}(3.8 \mathrm{a})\right.}_{\widetilde{R}_{12}-R_{12}} \\
& \left.H_{z}=\left(-\frac{i I A}{4 \pi}\right) \int_{0}^{\infty} d k_{\rho} \frac{k_{\rho}^{3}}{k_{1 z}} J_{o}\left(k_{\rho} \rho\right)\left[\frac{T_{12} \widetilde{R}_{23} T_{21} e^{i k_{2 z}\left(d_{2}-d_{1}\right)}}{1+R_{12} \widetilde{R}_{23} e^{i k_{2 z}\left(d_{2}-d_{1}\right)}}\right] e^{i k_{1 z}\left(z+z^{\prime}+2 \mathrm{~b}_{3}\right)} .8 \mathrm{~b}\right)
\end{aligned}
$$

Similarly, the Fréchet derivatives which contain two Green's functions that model induction current and scattering respectively are computed by the following expressions:

$$
\begin{aligned}
F_{m}\left(\rho, z, z^{\prime}\right)= & F_{m x}\left(\rho, z, z^{\prime}\right)-F_{m z}\left(\rho, z, z^{\prime}\right) \sin (\theta) \\
F_{m x}= & -\frac{i I A}{8 \pi} \int_{0}^{\infty} d k_{\rho} \frac{k_{\rho}^{2}}{k_{1 z}} J_{1}\left(k_{\rho} \rho\right) \widetilde{T}_{1 m} \widetilde{T}_{m 1} I_{m}\left(k_{\rho}\right) e^{i k_{1 z}\left(z+z^{\prime}\right)} \\
F_{m z}= & \frac{I A}{8 \pi} \int_{0}^{\infty} d k_{\rho} \frac{k_{\rho}^{3}}{k_{m z} k_{1 z}} J_{o}\left(k_{\rho} \rho\right) \widetilde{T}_{1 m} \widetilde{T}_{m 1} I_{m}\left(k_{\rho}\right) e^{i k_{1 z}\left(z+z^{\prime}\right)} \\
I_{m}\left(k_{\rho}\right)= & \frac{e^{2 i k_{m z} d_{m}}-e^{2 i k_{m z} d_{m-1}}}{2 i k_{m z}}\left(1+\widetilde{R}_{m, m+1}^{2}\left(k_{\rho}\right) e^{2 i k_{m z}\left(d_{m}-d_{m-1}\right)}\right) \\
& +2 \widetilde{R}_{m, m+1}\left(k_{\rho}\right) e^{2 i k_{m z} d_{m}}\left(d_{m}-d_{m-1}\right),
\end{aligned}
$$

where we note that $\mu_{1} \frac{\widetilde{T}_{1 m}}{k_{1 z}}=\mu_{m} \frac{\widetilde{T}_{m 1}}{k_{m z}}$ by reciprocity. Here, $I_{m}\left(k_{\rho}\right)$ is obtained by an analytical integration of the $z^{\prime \prime}$-dependent portion of (3.6), which is possible since each layer is assumed to be homogeneous.

The Sommerfeld integrals are rapidly computed by a combination of GaussLegendre and Gauss-Laguerre quadratures. Singularities are encountered in (3.8b), (3.10) and (3.11). However, these singularities are easily removed by extracting them from corresponding transmission coefficients; e.g, $\frac{T_{12}}{k_{1 z}}=$ $\frac{2}{k_{1 z}+k_{2 z}}$. The decaying tails of the integrands are integrated by Gauss-Laguerre quadrature which exploits its low frequency behavior, viz., $i k_{z} \approx-k_{\rho}$ for $k_{\rho} \gg k$. To be able to utilize this quadrature in the Fréchet derivatives even when the transmitter and receiver are at the same level, $z=z^{\prime}=0$, we note that each of the generalized transmission coefficients, $\widetilde{T}_{1 m}$ and $\widetilde{T}_{m 1}$, has an 
$e^{i k_{1 z} d_{1}}$ factor, which leads to the same exponential factor as in (3.8a) and (3.8b).

$$
\begin{aligned}
\int_{0}^{\infty} d k_{\rho} k_{\rho}^{n} f\left(k_{\rho}\right) e^{i k_{z}\left(z+z^{\prime}+2 d_{1}\right)} & =\int_{0}^{\alpha k} d k_{\rho} k_{\rho}^{n} f\left(k_{\rho}\right) e^{i k_{z}\left(z+z^{\prime}+2 d_{1}\right)}+ \\
\approx \underbrace{\int_{0}^{\alpha k} d k_{\rho} k_{\rho}^{n} f\left(k_{\rho}\right) e^{i k_{z}\left(z+z^{\prime}+2 d_{1}\right)}}_{\text {Gauss-Legendre }}+\underbrace{\int_{\alpha k}^{\infty} d k_{\rho} k_{\rho}^{n} f\left(k_{\rho}\right) e^{i k_{z}\left(z+z^{\prime}+2 d_{1}\right)}}_{\text {Gauss-Laguerre }} & \underbrace{}_{\text {- } d k_{\rho}^{\prime}\left[k_{\rho}^{\prime}+\alpha k\right]^{n} f\left(k_{\rho}^{\prime}+\alpha k\right) e^{-\left(k_{\rho}^{\prime}+\alpha k\right)\left(z+z^{\prime}+2 d_{1}\right)}}
\end{aligned}
$$

The guided mode poles given by the roots of the guidance condition

$$
1+R_{12} \widetilde{R}_{23} e^{i k_{2 z}\left(d_{2}-d_{1}\right)}=0
$$

can also be circumvented by deforming the integration path slightly below the real axis. For the relevant low frequencies, 25-point Gauss-Legendre and 15-point Gauss-Laguerre quadrature is adequate and readily yields the required performance. In fact, the recursive computation of reflection and transmission coefficients once for each frequency is the most time-consuming step in a DBIM iteration.

To solve the $1 \mathrm{D}$ inverse problem, we seek the minimum of the functional

$$
\mathcal{I}=\left|\sum_{i=1}^{N}\left[\Delta H^{i}(\rho, z)-\sum_{m=3}^{M} \delta \epsilon_{m} F_{m}^{\epsilon i}\left(\rho, z, z^{\prime}\right)-\sum_{m=3}^{M} \delta \sigma_{m} F_{m}^{\sigma i}\left(\rho, z, z^{\prime}\right)\right]\right|^{2}
$$

where $N$ frequencies are employed in the reconstruction and $F_{m}^{\epsilon}=k_{o}^{2} F_{m}$ and $F_{m}^{\sigma}=i k_{o} \eta_{o} F_{m}$. A subsequent discretization, expansion, and minimization yields

$$
\left[\begin{array}{ll}
\overline{\mathbf{F}}^{\epsilon \dagger} \cdot \overline{\mathbf{F}}^{\epsilon}+\gamma_{\epsilon} \overline{\mathbf{D}}^{\dagger} \cdot \overline{\mathbf{D}} & \overline{\mathbf{F}}^{\epsilon \dagger} \cdot \overline{\mathbf{F}}^{\sigma} \\
\overline{\mathbf{F}}^{\sigma \dagger} \cdot \overline{\mathbf{F}}^{\epsilon} & \overline{\mathbf{F}}^{\sigma \dagger} \cdot \overline{\mathbf{F}}^{\sigma}+\gamma_{\sigma} \overline{\mathbf{D}}^{\dagger} \cdot \overline{\mathbf{D}}
\end{array}\right]\left[\begin{array}{c}
\boldsymbol{\delta} \boldsymbol{\epsilon} \\
\boldsymbol{\delta} \boldsymbol{\sigma}
\end{array}\right]=\left[\begin{array}{c}
\overline{\mathbf{F}}^{\epsilon \dagger} \cdot \Delta \mathbf{H} \\
\overline{\mathbf{F}}^{\sigma \dagger} \cdot \Delta \mathbf{H}
\end{array}\right]
$$

in which $\gamma_{\epsilon}$ and $\gamma_{\sigma}$ are Tikhonov regularization parameters and $\overline{\mathbf{D}}$ is the regularization matrix. The regularization parameters are chosen sufficiently large 
to filter out high-frequency profiles but no larger to avoid unnecessary iterations. We have employed the same regularization parameters for simulation and real data processing.

We define the object function as $\tilde{\mathbf{O}}=[\boldsymbol{\delta} \boldsymbol{\epsilon}, \boldsymbol{\delta} \boldsymbol{\sigma}]^{t}$ and let

$$
\overline{\mathbf{M}}=\left[\begin{array}{cc}
\overline{\mathbf{F}}^{\epsilon} & \overline{\mathbf{F}}^{\sigma} \\
\sqrt{\gamma_{\epsilon}} \overline{\mathbf{D}} & \overline{\mathbf{0}} \\
\overline{\mathbf{0}} & \sqrt{\gamma_{\sigma}} \overline{\mathbf{D}}
\end{array}\right] .
$$

We also define a Hermitian weighting matrix $\overline{\mathbf{A}}$, which is utilized when lower frequencies are more reliable for the initial iterations, especially when considerably wideband frequency data is involved. With these definitions, (3.15) can be written in the more succinct form

$$
\underbrace{\overline{\mathbf{M}}^{\dagger} \cdot\left[\begin{array}{ccc}
\overline{\mathbf{A}} & \overline{\mathbf{0}} & \overline{\mathbf{0}} \\
\overline{\mathbf{0}} & \overline{\mathbf{I}} & \overline{\mathbf{0}} \\
\overline{\mathbf{0}} & \overline{\mathbf{0}} & \overline{\mathbf{I}}
\end{array}\right]}_{\overline{\mathbf{M}}^{\prime \dagger}} \cdot \overline{\mathbf{M}} \cdot \tilde{\mathrm{O}}=\overline{\mathbf{M}}^{\prime \dagger} \cdot\left[\begin{array}{c}
\Delta \mathbf{H} \\
\overline{\mathbf{0}} \\
\overline{\mathbf{0}}
\end{array}\right] .
$$

This reveals that the linear system can be solved by the CG method, which improves computational complexity. The relevant forward and adjoint operators are respectively $\overline{\mathbf{M}}$ and $\overline{\mathbf{M}}^{\prime \dagger}$. Also, unless $\overline{\mathbf{A}}=\overline{\mathbf{I}}$, the $\mathrm{CG}$ norm needs to be redefined as

$$
\|\mathbf{a}\|=\mathbf{a}^{\dagger} \cdot\left[\begin{array}{ccc}
\overline{\mathbf{A}} & \overline{\mathbf{0}} & \overline{\mathbf{0}} \\
\overline{\mathbf{0}} & \overline{\mathbf{I}} & \overline{\mathbf{0}} \\
\overline{\mathbf{0}} & \overline{\mathbf{0}} & \overline{\mathbf{I}}
\end{array}\right] \cdot \mathbf{a} .
$$

The above procedure is then repeated by updating the inhomogeneous background and the associated Green's function using the reconstructed parameters [13-15]. Although DBIM will not converge for highly conductive scatterers like metals, the reconstruction can still track the location of the strong scatterer. This is depicted in Figure 3.8 where the contrast is 40 with respect to a $22-\mathrm{mS} / \mathrm{m}$ background. Finally, we note that the above can be easily extended so that permeability as well as complex permittivity is reconstructed provided the contrasts are small, i.e., the problem is not very nonlinear.

In principle, the above can be easily extended so that permeability as well as complex permittivity is reconstructed. However, the TE reflection coefficient involves permeability in a very nonlinear manner and the resulting linear system is considerably ill-conditioned unless the perturbations in permeability 

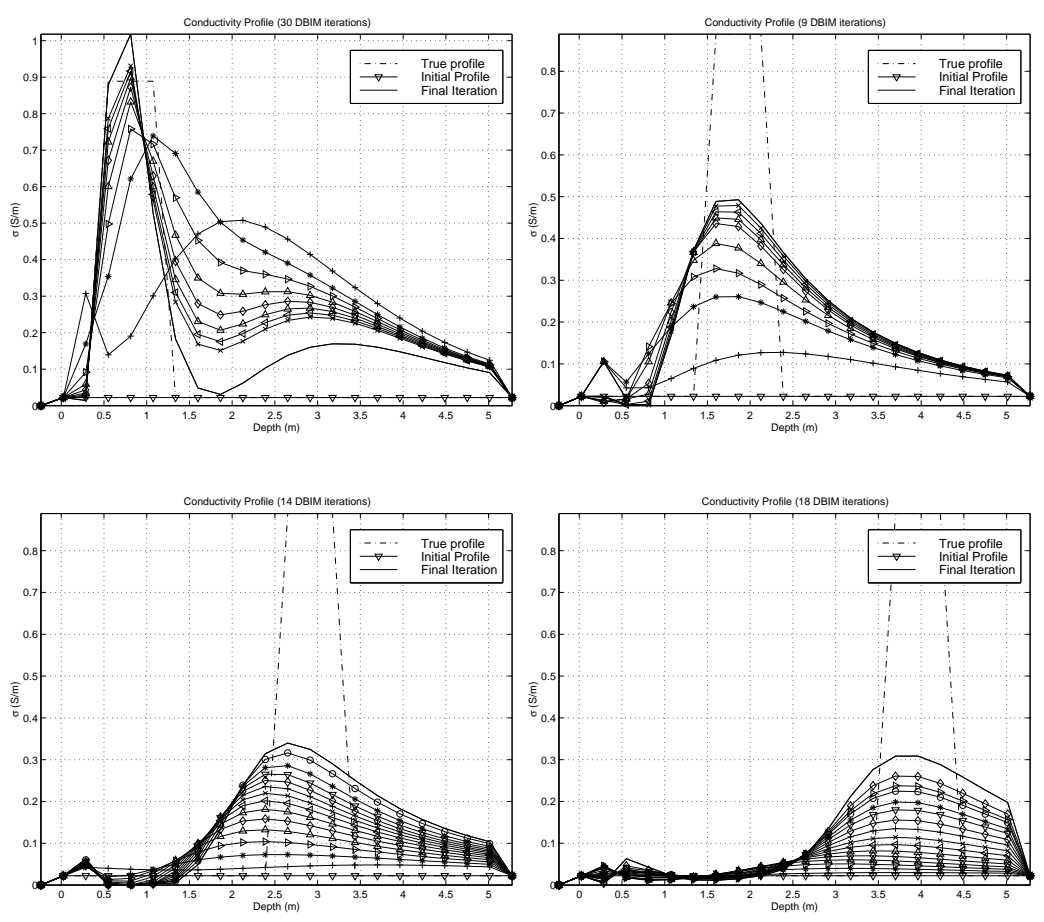

Figure 3.8: The 1D solver can track the location of strong scatterers. Contrast of the object is 40 with $\sigma_{b}=22 \mathrm{mS} / \mathrm{m}$. When the scatterer is shallow, DBIM manages to converge to the profile. When it is deeper, it tracks the position of the scatterer successfully but not the profile.

are small. The distorted Born approximation in (3.7) will still be able to linearize the problem if we let

$\delta k_{m}^{2}=\Delta\left(\mu_{m}\left[k_{o}^{2} \epsilon_{m}+i k_{o} \eta_{o} \sigma_{m}\right]\right) \approx \mu_{m_{o}} \Delta\left(k_{o}^{2} \epsilon_{m}+i k_{o} \eta_{o} \sigma_{m}\right)+\left[k_{o}^{2} \epsilon_{m_{o}}+i k_{o} \eta_{o} \sigma_{m_{o}}\right] \Delta \mu_{m}$

where the double subscripted parameters denote the parameters obtained by the previous DBIM iteration. Akin to (3.16), the overall Fréchet matrix becomes 


$$
\overline{\mathbf{M}}=\left[\begin{array}{ccc}
\overline{\mathbf{F}}^{\epsilon} \cdot \overline{\mathcal{D}}(\boldsymbol{\mu}) & \overline{\mathbf{F}}^{\sigma} \cdot \overline{\mathcal{D}}(\boldsymbol{\mu}) & \overline{\mathbf{F}}^{\mu} \\
\sqrt{\gamma_{\epsilon}} \overline{\mathbf{D}} & \overline{\mathbf{0}} & \overline{\mathbf{0}} \\
\overline{\mathbf{0}} & \sqrt{\gamma_{\sigma}} \overline{\mathbf{D}} & \overline{\mathbf{0}} \\
\overline{\mathbf{0}} & \overline{\mathbf{0}} & \sqrt{\gamma_{\mu}} \overline{\mathbf{D}}
\end{array}\right]
$$

Here $\overline{\mathbf{F}}^{\mu}$ is a matrix whose elements are established by multiplying the elements of $\overline{\mathbf{F}}$ with corresponding complex permittivities, $k_{o}^{2} \epsilon_{m_{o}}+i k_{o} \eta_{o} \sigma_{m_{o}}, \overline{\mathcal{D}}$ is an operator that converts a vector to a diagonal matrix, and the object function is $\tilde{\mathbf{O}}=[\boldsymbol{\delta} \boldsymbol{\epsilon}, \boldsymbol{\delta} \boldsymbol{\sigma}, \boldsymbol{\delta} \boldsymbol{\mu}]^{t}$. For large scale applications, we opted to employ the complex permittivity inversion since it is more benign and computationally less intensive. More importantly, changes in permeability are rare in practice.

\subsubsection{Simulation of the 1D Solver in a 2D Environment}

It is well known that the 1D DBIM inverse solver can easily handle 1D profiles with contrasts less than 10 [16]. Yet it is crucial to know how it would behave in a $2 \mathrm{D}$ or $3 \mathrm{D}$ environment to ensure its viability as a fast probing tool. For this purpose, 11 transmitter-receiver pairs and 11 frequencies have been simulated by using a 2D TM solver.

The contrast in Figure 3.9 is 20 with respect to a background of $5 \mathrm{mS} / \mathrm{m}$. Here, the 2-m spaced transmitter-receiver pair was simulated for 11 locations along a distance of $20 \mathrm{~m}$. At each point, 11 equally spaced frequencies from $0.5 \mathrm{MHz}$ to $5.5 \mathrm{MHz}$ were employed in the inversion. It is seen that the horizontal resolution for the deepest object is not as good as the others due to the diffusion of the field. However, the depth information for each is approximately correct. Figure 3.10 illustrates a case where the 1D solver forms a faded false image between the two actual scatterers. This is because the scattering from the two objects adds constructively. Essentially, the 1D solver is totally unaware of a second multiple scattering dimension.

Although even more multiple scattering in 3D exacerbates this problem, we note that false images have lower contrasts than those of actual images and that they form deep in the profile.

\subsubsection{Simulation of the 1D Solver in a 3D Environment}

Here, we provide a $3 \mathrm{D}$ simulation of a $2 \mathrm{D}$ metal object to verify the background estimation and inversion methods. In this simulation, a VMD trans- 
mitter and a HMD receiver pair traverse a $7 \mathrm{~m} \times 7 \mathrm{~m}$ region. The receiver is tilted by $2^{o}$ toward the ground. At each point, a time domain measurement is made. The time domain waveform is obtained by using the frequency-domain solution of a 3D CGFFT solver performing 2D FFTs over the surface of a 2 $\mathrm{m} \times 2 \mathrm{~m}$ metal plate, two opposite corners of which are located at $(-1 \mathrm{~m},-1$ $\mathrm{m})$ and $(1 \mathrm{~m}, 1 \mathrm{~m})$ in the plots. The problem is solved for 1024 frequencies ranging from DC to $10 \mathrm{MHz}$ at each point for a total of 196 pixels.

Any sufficiently high-frequency noise in Figure 3.11 does not pose an insurmountable problem for the reconstruction because 11 low frequencies up to $3.0 \mathrm{MHz}$ are involved in the inversion. Because of the strong direct coupling in the opposite direction, the scattering from the metal plate actually decreases the magnitude and slope of the field before $2.0 \mu \mathrm{s}$ in the figure.

The background is estimated by using the latter method in Section 3.1.2. The estimated background at $5.0 \mathrm{MHz}$ is around $18 \mathrm{mS} / \mathrm{m}$, which approximates the actual $20 \mathrm{mS} / \mathrm{m}$ as shown in Figure 3.12. We do not employ higher frequencies for the estimation, mainly because the dipole approximation deteriorates and real data becomes a lot more noisy. The reconstruction detects the metal plate at a depth of around $1.2 \mathrm{~m}$. It is anticipated that the $1 \mathrm{D}$ solver would miss the multiple scattering at the corners of the plate and detect a plate larger than the actual one.

Similar results are obtained for two close scatterers with contrasts of 5:1 and $5: 2$ as shown in Figure 3.13. Here, the background conductivity is $10 \mathrm{mS} / \mathrm{m}$ and the simulated data is obtained by a 3D CGFFT electric flux forward solver. The corresponding reconstruction is shown in Figure 3.14. Because the receiver is collecting the near field with high spectral content, the objects can be superresolved although the smallest wavelength in the data is $100 \mathrm{~m}$ long. On the other hand, the higher contrast object appears to be shallower, possibly because the multiple scattering enhancement to field amplitude is accounted by a shallower layer.

\subsubsection{Application of 1D Inversion to Field Data}

The Denver Federal Center (DFC) is covered with mineralogical clay soils that are quite conductive. GPR results there have generally proved disappointing. East of the present Building 20 at the DFC, there is a dirt field approximately $40 \mathrm{~m}$ by $90 \mathrm{~m}$. The VETEM system was first set up and run at that location merely to demonstrate procedures to be used at another site. However, it was noticed that over the DFC field, VETEM produced strong 
and highly variable signals. We have since learned that the field is on the site of a former WWII munitions foundry. Although the foundry building is gone, many buried subsurface structures remain [17].

Although amplitude plots can help locate objects, they do not yield much information concerning the depth of objects. Figures 3.15 and 3.16 contain plots of our reconstruction, which clearly identifies many chambers and hallways. It also compares well with the amplitude shaded relief in [17]. At each measurement location, the depth where conductivity reconstruction attains a maximum has been sampled to generate this plot. The site consists of 17,665 waveforms each consisting of 8,192 time domain samples of the receiver response digitized as a 4 -byte integer. Including coordinate information, this corresponds to over 250 Mbytes of data, which took around 3 hours of user time to process on a $650-\mathrm{MHz}$ Alpha workstation. This particular reconstruction was done with 22 layers each 25 -cm thick and 11 frequencies ranging from $60 \mathrm{kHz}$ to $3.05 \mathrm{MHz}$.

The 1D inversion solver is also used to process the VETEM data collected at DOE Pit 9. The results is shown Figure 3.17.

\subsubsection{Computational Complexity}

The method has the modest memory requirements of a 1D inverse solver. Hence, we will solely study computational complexity, which is predominantly due to the inverse solver rather than due to the calibration process. Calibration consists of an FFT, background estimation by sampling the waveform slope, deconvolution of the system function and two Sommerfeld integrations for each waveform. Hence, the complexity of calibration is $N_{w} \times\left[c_{1} O\left(N_{s} \log \left(N_{s}\right)\right)+c_{2}\right]$, where $N_{w}$ is the number of waveforms and $N_{s}$ is the number of time domain samples.

Because the number of DBIM iterations is contingent upon the strength and shape of the scatterer, the complexity of the 1D solver which also depends on the number of Sommerfeld integrals can be expressed as $O\left(\sum_{i=1}^{N_{w}} N_{i} N_{l} N_{f}\right)$. Here, $N_{i}$ is the number of DBIM iterations for the $i$ th waveform, $N_{l}$ is the number of layers, and $N_{f}$ is the number of frequencies. Typically, $N_{l}$ and $N_{f}$ are small, which renders the overhead of the linear equation solver negligible. The basic strength of this method is that computational complexity increases linearly with the size of the scanned area, i.e., $N_{w}$. 

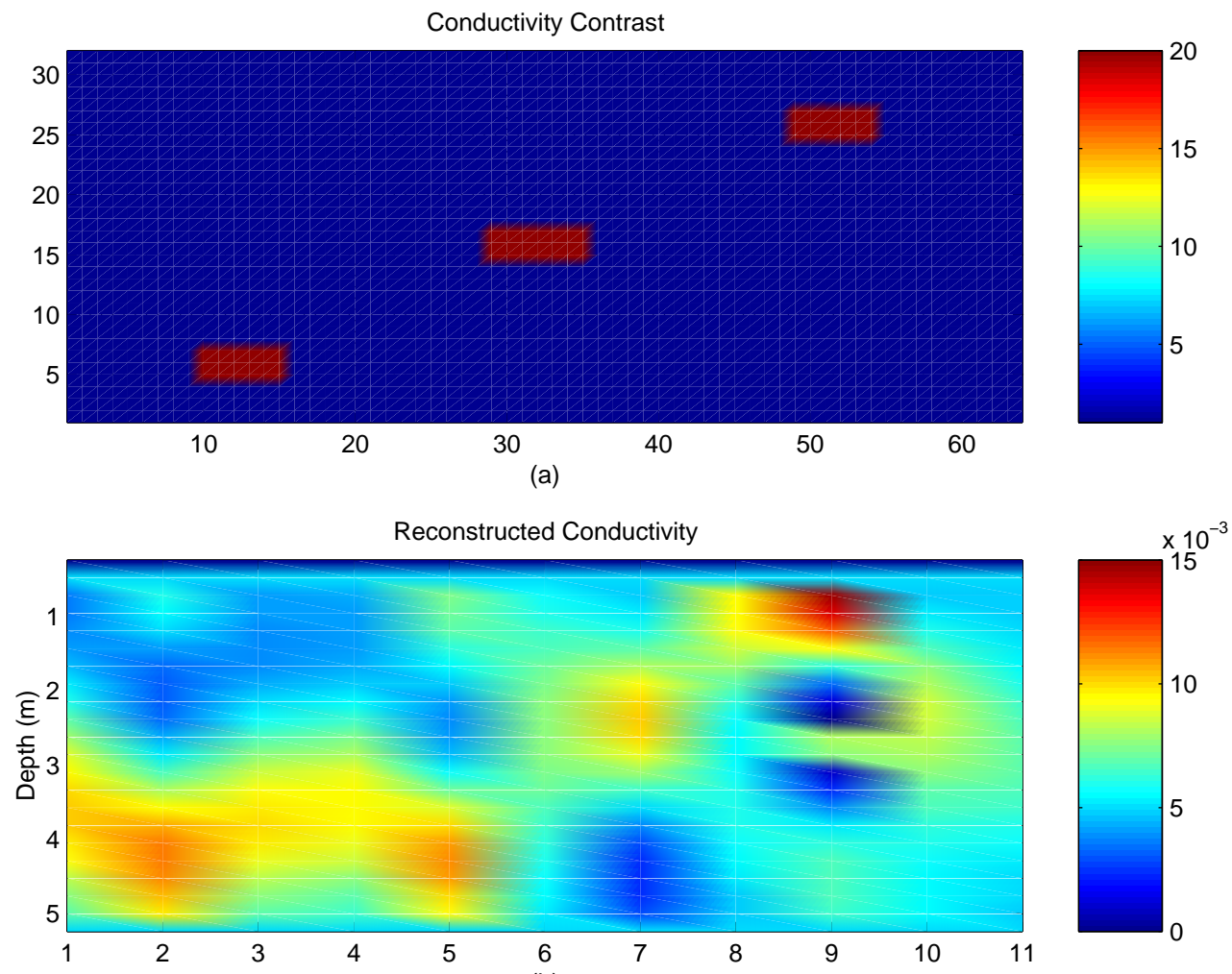

(b)

Figure 3.9: The 1D solver in a $2 \mathrm{D}$ environment. (a) Profile input to the $2 \mathrm{D}$ TM MoM solver. Discretization is $64 \times 32$ pixels for $20 \times 5 \mathrm{~m} . \sigma_{b}=5 \mathrm{mS} / \mathrm{m}$. (b) Although the line-source code fails to converge, it locates the objects with only 11 frequencies, $0.5-5.5 \mathrm{MHz}$. The 2-m spaced transmitter-receiver pair was simulated for 11 locations along a distance of $20 \mathrm{~m}$. Final conductivity rather than contrast is shown. 

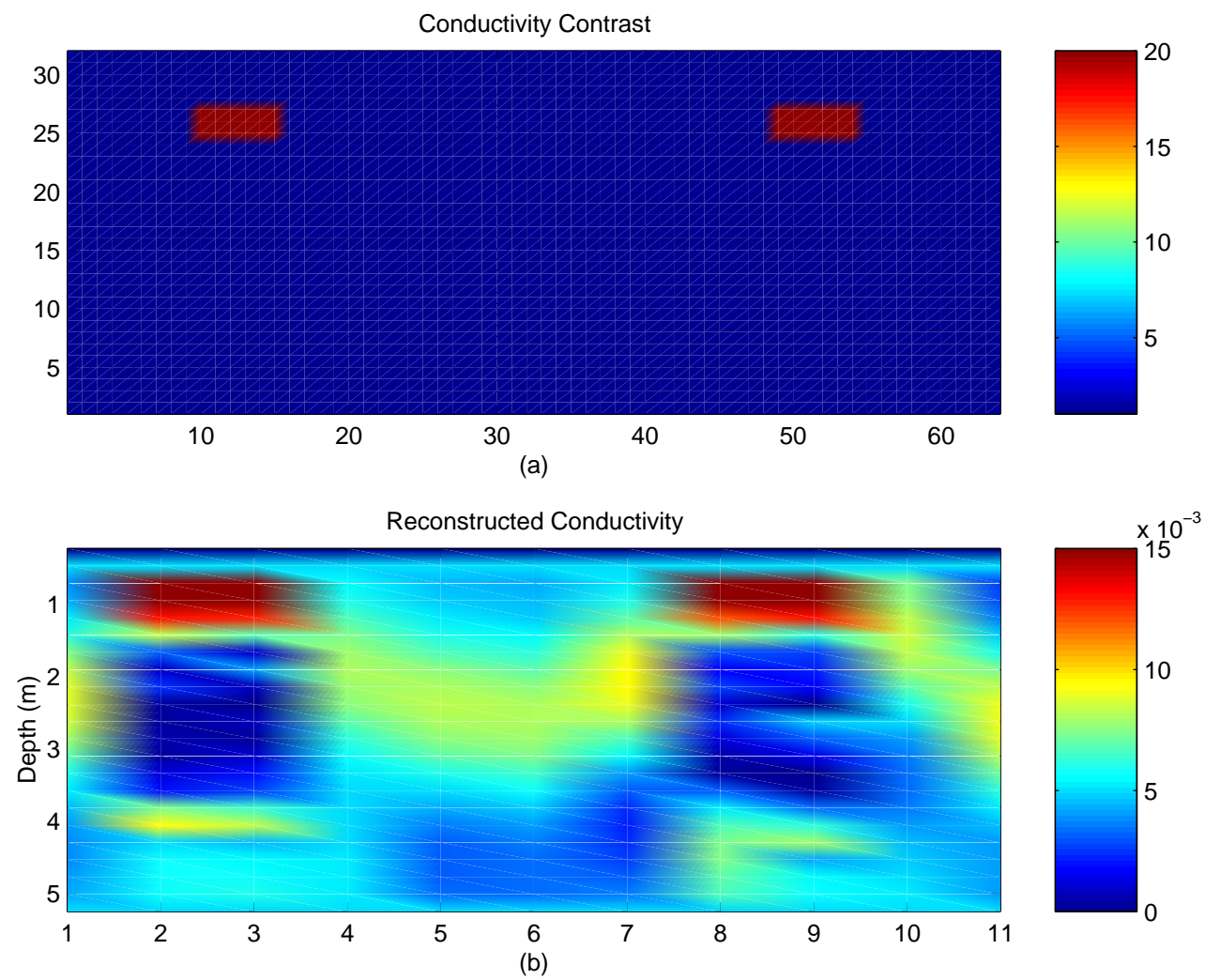

Figure 3.10: (a) Profile input to the 2D TM MoM solver. Discretization is $64 \times$ 32 pixels for $20 \times 5 \mathrm{~m}$. $\sigma_{b}=5 \mathrm{mS} / \mathrm{m}$. (b) A false image forms between the two scatterers. The transmitter-receiver pair was simulated for 11 locations that are evenly spaced along $20 \mathrm{~m}$. Final conductivity rather than contrast is shown. 


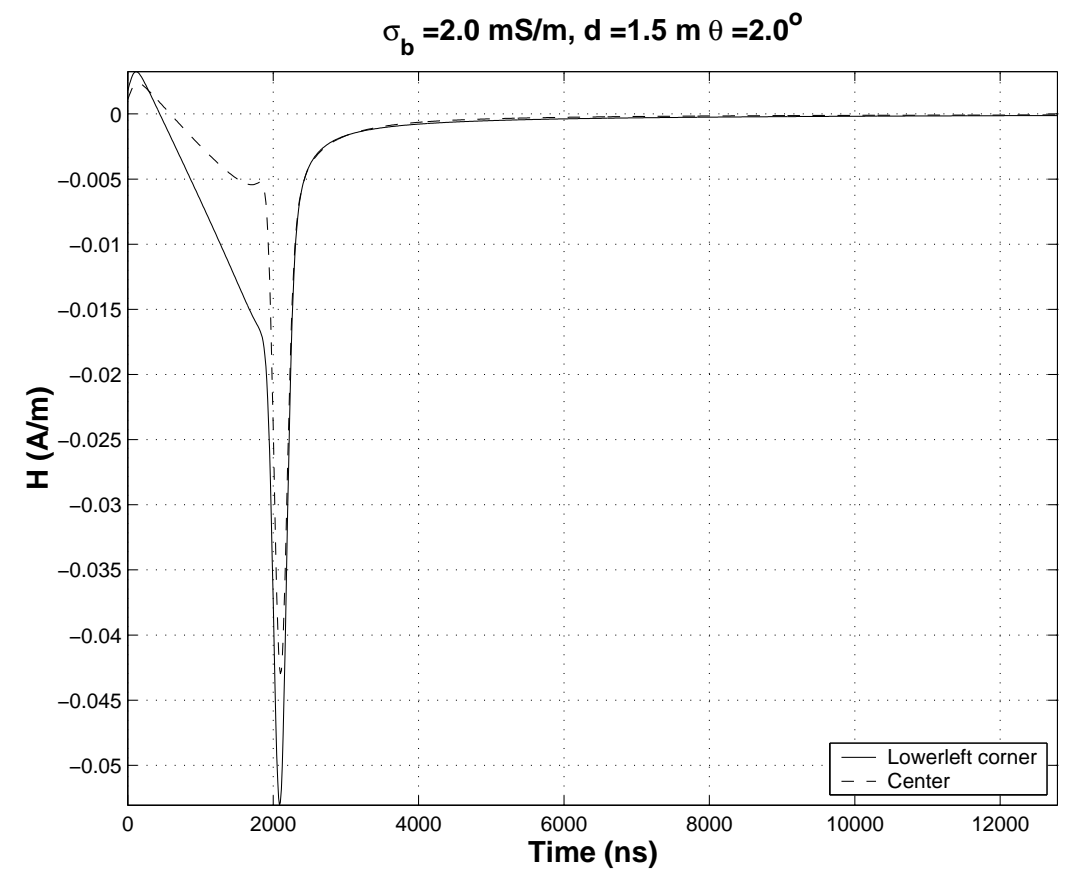

Figure 3.11: Simulated waveforms right above the PEC plate and far away. 

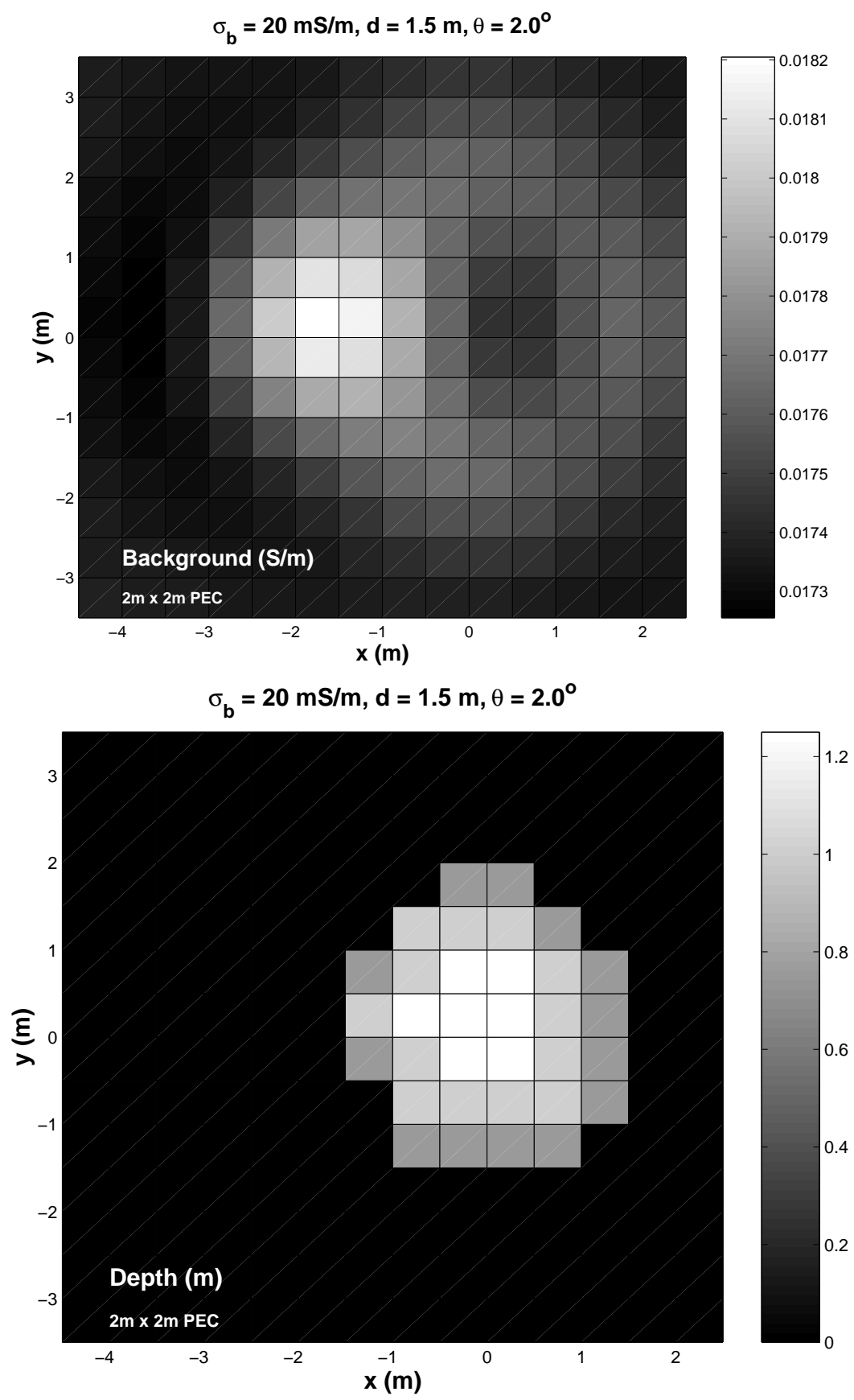

Figure 3.12: Estimated (a) background and (b) depth. 

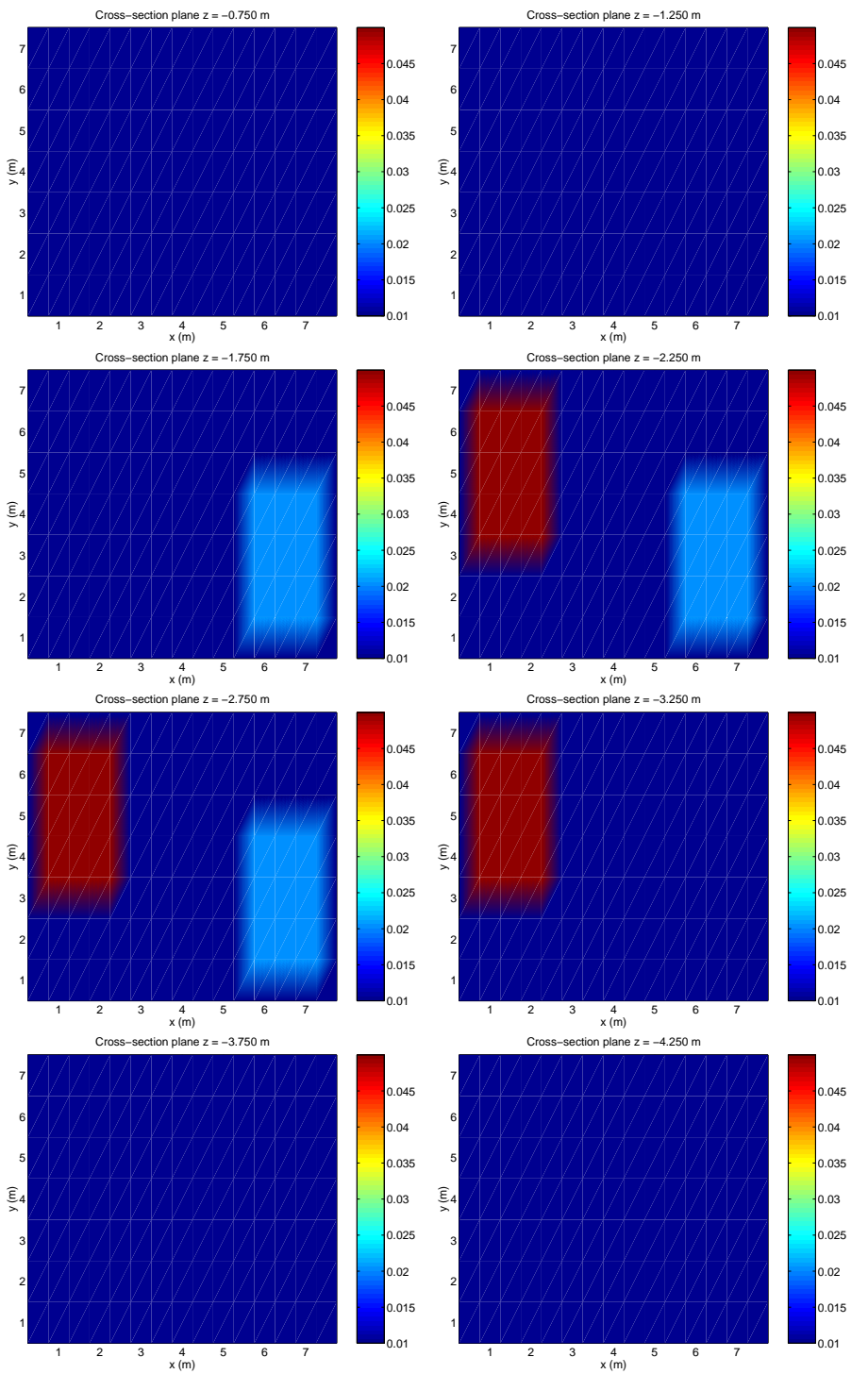

Figure 3.13: True object profile at different depth cross sections. 


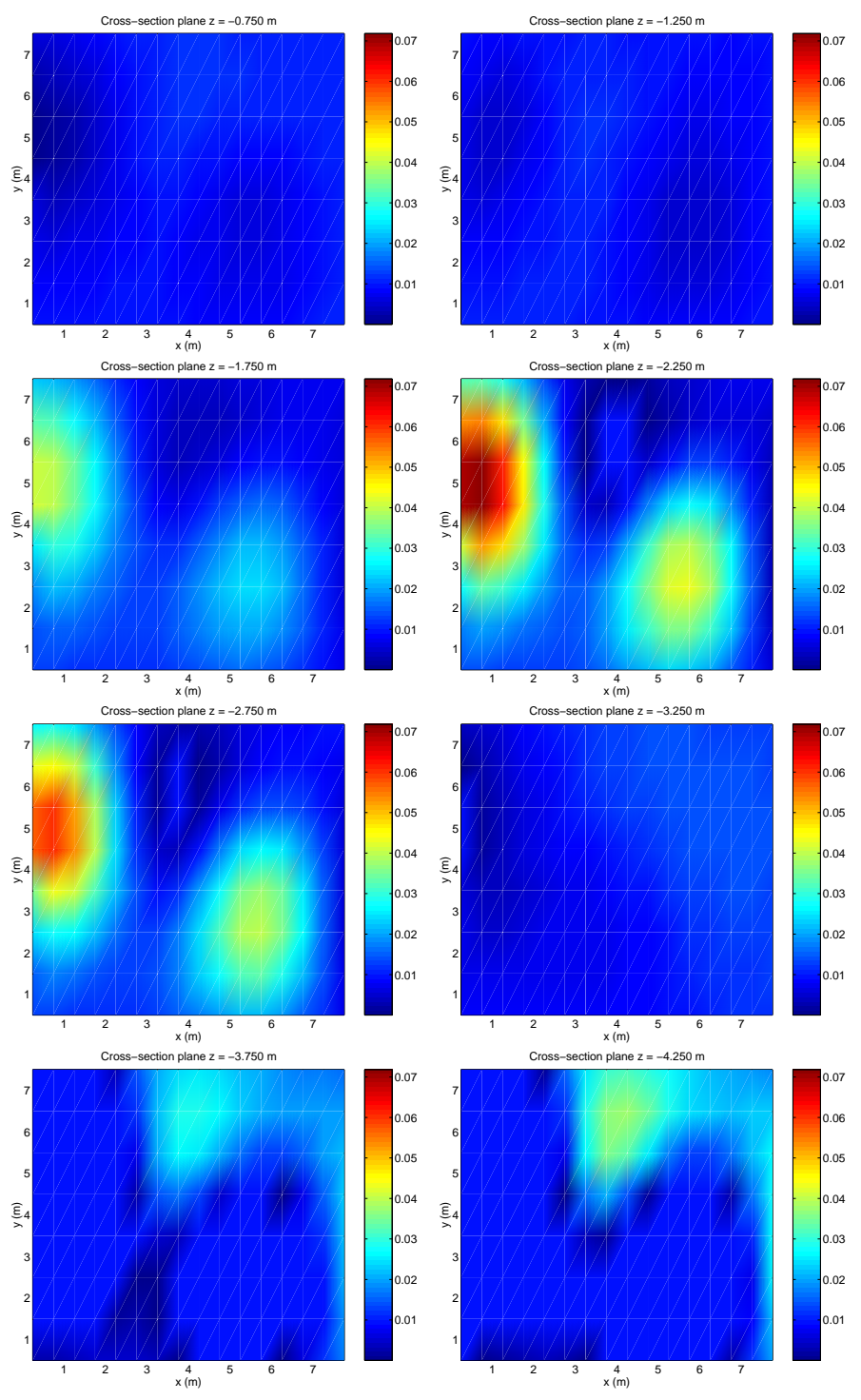

Figure 3.14: Reconstructed images at different depth cross sections. 


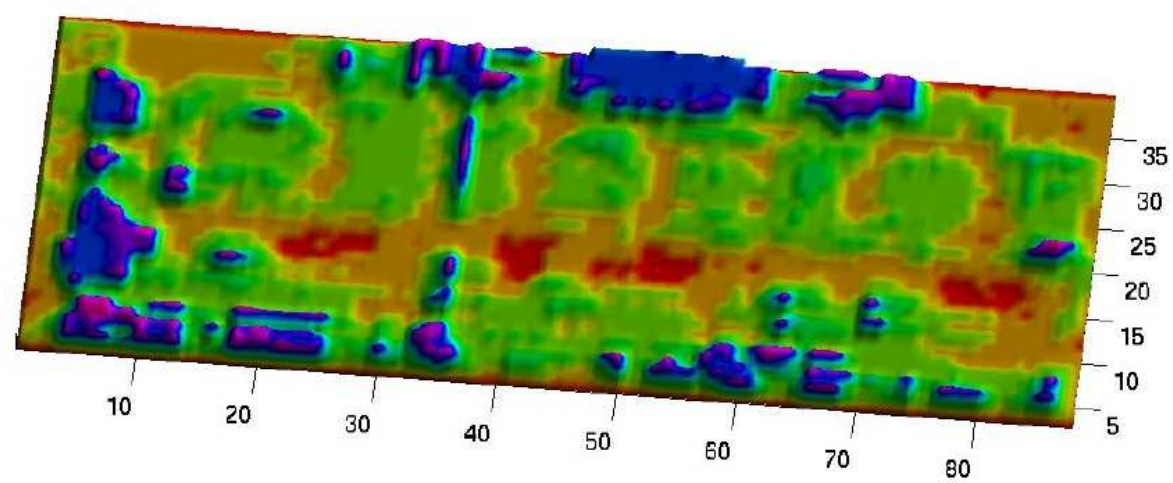

Figure 3.15: A relief of the substructures at the foundry site.

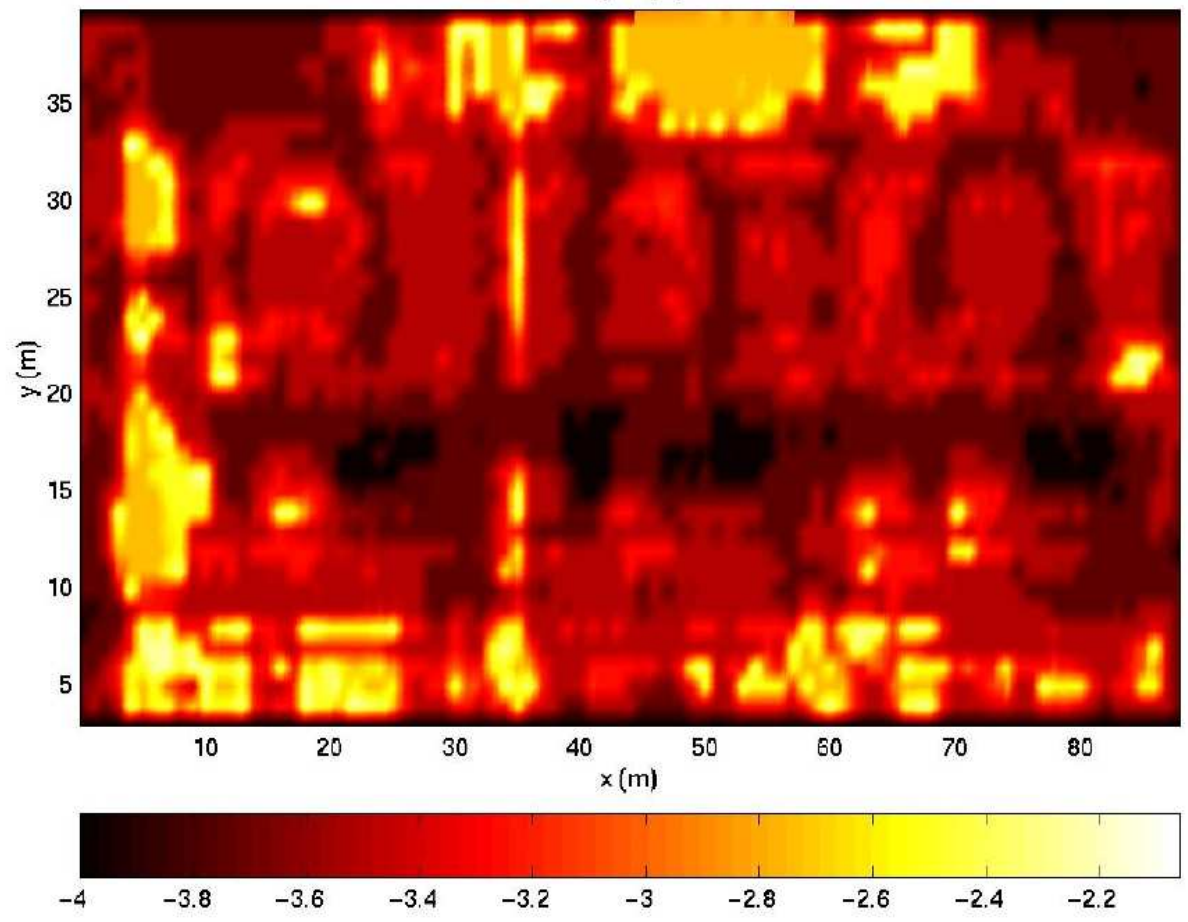

Figure 3.16: Depth of the substructures at the foundry site. 


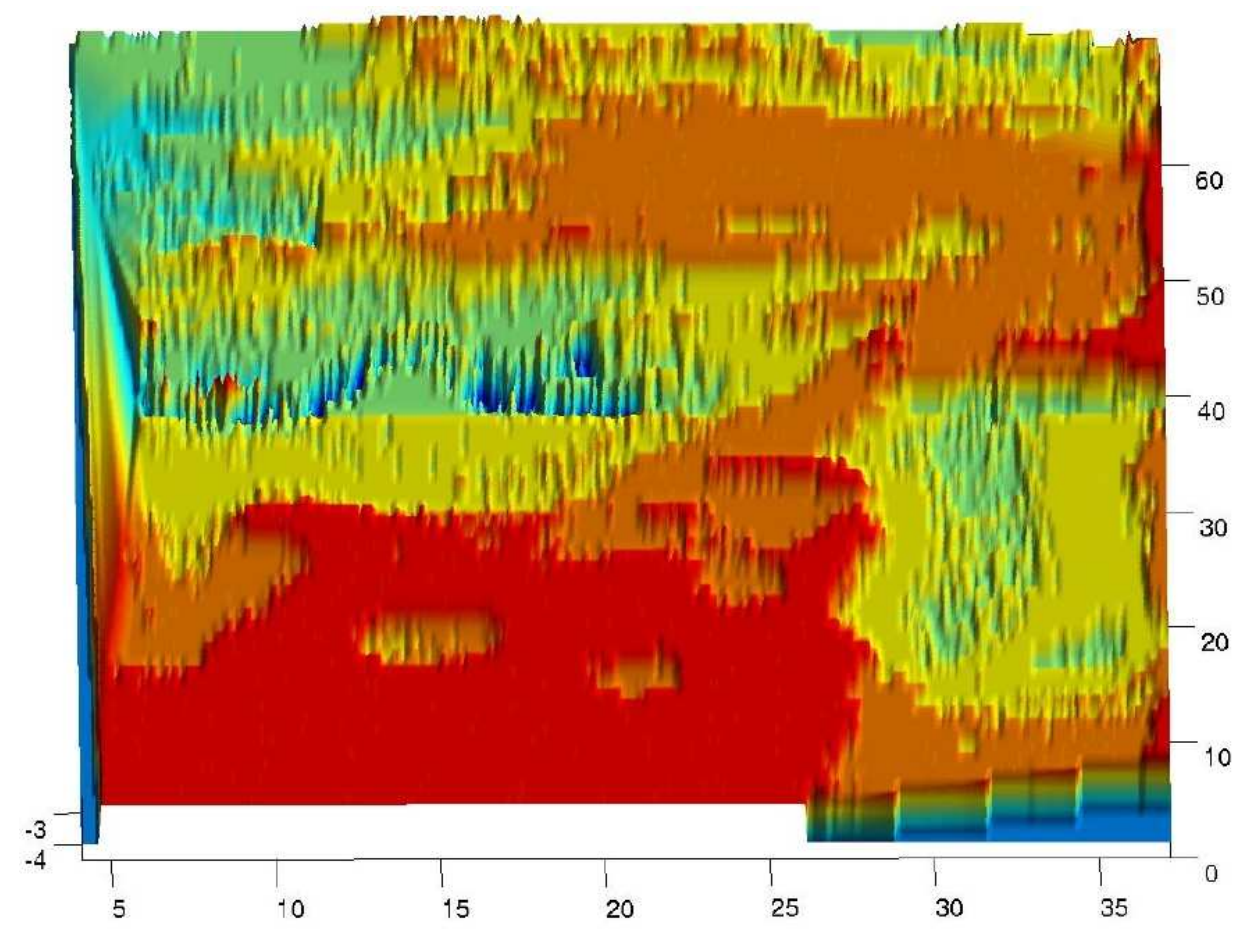

Figure 3.17: The 1D DBIM relief image of the DOE Pit-9 site. The depths of the buried objects range from $2.5 \mathrm{~m}$ to $4.0 \mathrm{~m}$. 


\subsection{D Inversion by the Born Approximation and the Distorted Born Iterative Method}

In this section, we present the 3D inversion algorithm with DBIM [18]. The Born approximation can be thought of as a special case of DBIM, therefore will not be introduced separately.

\subsubsection{Half-space Volume Integral Equation}

An electric flux, $\mathbf{D}$, formulation is used to cope with the large variations in complex permittivity since the alternative discretization of $\mathbf{J}$ jumps across conductivity discontinuities.

For the dielectric object buried under the ground surface, the volume integral equation (VIE) can be written as

$$
\mathbf{E}^{i n c}(\mathbf{r})=\frac{\varepsilon_{b}}{\varepsilon_{r}(\mathbf{r})} \widetilde{\mathbf{D}}(\mathbf{r})-k_{b}^{2} \int_{V} d \mathbf{r}^{\prime}\left[\overline{\mathbf{G}}_{b}^{P}\left(\mathbf{r}, \mathbf{r}^{\prime}\right)+\overline{\mathbf{G}}_{b}^{R}\left(\mathbf{r}, \mathbf{r}^{\prime}\right)\right] \cdot \chi\left(\mathbf{r}^{\prime}\right) \widetilde{\mathbf{D}}\left(\mathbf{r}^{\prime}\right)
$$

where the subscript $b$ denotes the ground half-space; the superscripts $P, R$ represent the primary and reflected fields respectively; and $\varepsilon_{b}, \varepsilon_{r}(\mathbf{r})$ are the relative permittivities of the earth and the buried object. Here, $\mathbf{D}(\mathbf{r})$ and $\chi(\mathbf{r})$ are defined as

$$
\widetilde{\mathbf{D}}(\mathbf{r})=\frac{\mathbf{D}(\mathbf{r})}{\varepsilon_{b} \varepsilon_{0}}, \chi(\mathbf{r})=\frac{\varepsilon_{r}(\mathbf{r})-\varepsilon_{b}}{\varepsilon_{r}(\mathbf{r})} .
$$

The Green's functions are given by

$$
\mathbf{G}_{b}^{P}\left(\mathbf{r}, \mathbf{r}^{\prime}\right)=\left[1+\frac{\nabla \nabla}{k_{b}^{2}}\right] g^{P}\left(\mathbf{r}, \mathbf{r}^{\prime}\right)
$$

and

$$
\begin{gathered}
\mathbf{G}_{b}^{R}\left(\mathbf{r}, \mathbf{r}^{\prime}\right)=(\hat{x} \hat{x}+\hat{y} \hat{y}) g_{T E}^{R}+\hat{z} \hat{z} g_{T M}^{R}+\frac{1}{k_{b}^{2}}\left[\nabla_{s} \nabla_{s} g_{E M 1}^{R}\left(\mathbf{r}, \mathbf{r}^{\prime}\right)-\right. \\
\left.\hat{z} \frac{\partial}{\partial z} \nabla_{s} g_{T M}^{R}\left(\mathbf{r}, \mathbf{r}^{\prime}\right)+\nabla \frac{\partial}{\partial z} \hat{z} g_{T M}^{R}\left(\mathbf{r}, \mathbf{r}^{\prime}\right)\right]
\end{gathered}
$$

In the above, $g^{P}\left(\mathbf{r}, \mathbf{r}^{\prime}\right)$ is the scalar Green's function for the primary field in the earth 


$$
g^{P}\left(\mathbf{r}, \mathbf{r}^{\prime}\right)=\frac{e^{i k_{b}\left|\mathbf{r}-\mathbf{r}^{\prime}\right|}}{4 \pi\left|\mathbf{r}-\mathbf{r}^{\prime}\right|},
$$

and $g_{T E}^{R}\left(\mathbf{r}, \mathbf{r}^{\prime}\right), g_{T M}^{R}\left(\mathbf{r}, \mathbf{r}^{\prime}\right)$, and $g_{E M 1}^{R}\left(\mathbf{r}, \mathbf{r}^{\prime}\right)$ are the scalar Green's functions for the reflected field in the earth. The latter are

$$
g_{T E, T M, E M 1}^{R}\left(\mathbf{r}, \mathbf{r}^{\prime}\right)=\frac{i}{8 \pi^{2}} \int_{-\infty}^{+\infty} \int_{-\infty}^{+\infty} d \mathbf{k}_{s} \frac{R^{T E, T M, E M 1}}{k_{b z}} e^{i \mathbf{k}_{s}\left|\mathbf{r}_{s}-\mathbf{r}_{s}^{\prime}\right|-i k_{b z}\left(z+z^{\prime}\right)},
$$

where $R^{T E}$ and $R^{T M}$ are the reflection coefficients for TE wave and TM wave from region $b$, the earth half-space, to region $a$, the air half-space. The reflection coefficient $R^{E M 1}$ is defined as $\frac{k_{b}^{2}}{k_{s}^{2}} R^{T E}+\frac{k_{b z}^{2}}{k_{s}^{2}} R^{T M} . k_{b}=k_{0} \sqrt{\varepsilon_{b}}$ is the wave number in the earth. $k_{b z}$ is its vertical component, $k_{b z}=\sqrt{k_{b}^{2}-k_{s}^{2}}$. $k_{s}^{2}=k_{x}^{2}+k_{y}^{2} \cdot \nabla_{s}=\hat{x} \partial_{x}+\hat{y} \partial_{y}, \mathbf{k}_{s}=\hat{x} k_{x}+\hat{y} k_{y}$, and $\mathbf{r}_{s}=\hat{x} x+\hat{y} y$.

Using the weak-form discretization in [19] to transform (3.21) into its linear system counterpart, we obtain seven integrals to compute the magnetic potentials by the induced current. Three of these are related to the induction terms

$$
\begin{gathered}
A_{s s}^{i}=\int_{V} d \mathbf{r}^{\prime}\left[g^{P}\left(\mathbf{r}, \mathbf{r}^{\prime}\right)+g_{T E}^{R}\left(\mathbf{r}, \mathbf{r}^{\prime}\right)\right] \chi\left(\mathbf{r}^{\prime}\right) \widetilde{D}_{s}, \quad s=x, y \\
A_{z z}^{i}=\int_{V} d \mathbf{r}^{\prime}\left[g^{P}\left(\mathbf{r}, \mathbf{r}^{\prime}\right)+g_{T M}^{R}\left(\mathbf{r}, \mathbf{r}^{\prime}\right)\right] \chi\left(\mathbf{r}^{\prime}\right) \widetilde{D}_{z} .
\end{gathered}
$$

The others are due to the charge terms

$$
\begin{array}{r}
A_{u v}^{c}=\int_{V} d \mathbf{r}^{\prime}\left[g^{P}\left(\mathbf{r}, \mathbf{r}^{\prime}\right)+g_{E M 1}^{R}\left(\mathbf{r}, \mathbf{r}^{\prime}\right)\right] \chi\left(\mathbf{r}^{\prime}\right) \widetilde{D}_{v}, \quad u, v=x, y \\
A_{z s}^{c}=\int_{V} d \mathbf{r}^{\prime}\left[g^{P}\left(\mathbf{r}, \mathbf{r}^{\prime}\right)-g_{T M}^{R}\left(\mathbf{r}, \mathbf{r}^{\prime}\right)\right] \chi\left(\mathbf{r}^{\prime}\right) \widetilde{D}_{s}, \quad s=x, y .
\end{array}
$$

The components $A_{u z}^{c}, u=x, y, z$ are the same as $A_{z z}^{i}$ and are therefore obtained directly from (3.28). For the primary field, the above integrals are convolutions of the respective Green's functions with the induced currents; whereas for the reflected fields, they are convolutions in the $x$ and $y$ directions and correlations in the $z$ direction $[20,21]$.

The iterative method used to solve the resulting linear system is the stabilized biconjugate gradient (Bi-CGSTAB) method [22], also referred to as BCGS 
for brevity in literatures. BCGS generally converges more rapidly than the conjugate gradient ( $\mathrm{CG}$ ) and biconjugate gradient (BiCG) methods.

\subsubsection{D Half-space Distorted Born Iterative Method}

We use the complex conductivity instead of the complex permittivity because conduction currents are predominant over induction currents for the VETEM frequency range. The complex conductivity is defined as

$$
\tilde{\sigma}=\sigma-i \omega \varepsilon_{r} \varepsilon_{0}
$$

where the tilde is used to discriminate between the complex conductivity and the real conductivity. In the following, the tilde will be dropped for simplicity; therefore, the conductivity will be of complex form implicitly.

Once the induced current is already known, the scattered magnetic field is obtained by

$$
\mathbf{H}^{s}(\mathbf{r}) \cdot \hat{\alpha}_{R}=\hat{\alpha}_{R} \cdot \int_{V} d \mathbf{r}^{\prime} \nabla \times \overline{\mathbf{G}}_{e}\left(\mathbf{r}, \mathbf{r}^{\prime}\right) \delta \sigma\left(\mathbf{r}^{\prime}\right) \cdot \mathbf{E}\left(\mathbf{r}^{\prime}\right),
$$

where $\hat{\alpha}_{R}$ is the polarization of the receiver in the VETEM system, and $\delta \sigma(\mathbf{r})$ is the conductivity perturbation with respect to the background. If the background is a half-space, $\nabla \times \overline{\mathbf{G}}_{e}$ is obtained by the evaluation of Sommerfeld integrals. In the ensuing iterations for which the background is inhomogeneous, $\nabla \times \overline{\mathbf{G}}_{e}$ is computed numerically by the BCGS-FFT method. The scattered magnetic field is the measurement data required for inversion. In the distorted Born iterative method, a successive linearization strategy is utilized. Suppose the conductivity profile obtained in the last iteration is $\sigma^{(l)}$, referring to Equation (3.32), and neglecting higher order terms, we have

$$
\delta \mathbf{H}^{s(l)}(\mathbf{r}) \cdot \hat{\alpha}_{R}=\hat{\alpha}_{R} \cdot \int_{V} d \mathbf{r}^{\prime} \nabla \times \overline{\mathbf{G}}_{e}^{(l)}\left(\mathbf{r}, \mathbf{r}^{\prime}\right) \delta \sigma^{(l+1)}\left(\mathbf{r}^{\prime}\right) \cdot \mathbf{E}^{(l)}\left(\mathbf{r}^{\prime}\right),
$$

where $\mathbf{E}^{(l)}$ is the total internal electric field, which is computed in the presence of the conductivity profile $\sigma^{(l)}$ obtained in the earlier iteration. Here, $\overline{\mathbf{G}}_{e}^{(l)}\left(\mathbf{r}, \mathbf{r}^{\prime}\right)$ is the dyadic Green's function in the conductivity profile $\sigma^{(l)}$. The incremental field $\delta \mathbf{H}^{s(l)}(\mathbf{r})$ is

$$
\delta \mathbf{H}^{s(l)}(\mathbf{r})=\mathbf{H}^{s}(\mathbf{r})-\mathbf{H}^{s(l)}(\mathbf{r}),
$$


where $\mathbf{H}^{s}\left(\mathbf{r}_{R}\right)$ is the scattered field due to the true conductivity profile, i.e., the measurement data, and $\mathbf{H}^{s(l)}\left(\mathbf{r}_{R}\right)$ is the scattered field due to the conductivity profile $\sigma^{(l)}$ computed after the internal field $\mathbf{E}^{(l)}$ is obtained. Explicitly,

$$
\mathbf{H}^{s(l)}(\mathbf{r}) \cdot \hat{\alpha}_{R}=\hat{\alpha}_{R} \cdot \int_{V} d \mathbf{r}^{\prime} \nabla \times \overline{\mathbf{G}}_{e}^{(0)}\left(\mathbf{r}, \mathbf{r}^{\prime}\right)\left[\sigma^{(l)}\left(\mathbf{r}^{\prime}\right)-\sigma^{(0)}\left(\mathbf{r}^{\prime}\right)\right] \cdot \mathbf{E}^{(l)}\left(\mathbf{r}^{\prime}\right),
$$

where $\overline{\mathbf{G}}_{e}^{(0)}\left(\mathbf{r}, \mathbf{r}^{\prime}\right)$ is the Green's function in the half-space, and $\sigma^{(0)}$ is the conductivity of the earth, $\sigma_{b}$.

Discretizing the support of the unknown object into a number of uniform pixels, and expanding the conductivity correction $\delta \sigma^{(l+1)}$ in terms of a pulse basis defined over the set of the pixels, we can write (3.33) as

$$
\mathbf{b}=\mathbf{K} \cdot \mathbf{a} .
$$

Here, $\mathbf{b}$ is the measurement data,

$$
\mathbf{b}=\hat{\alpha}_{R} \cdot\left[\delta \mathbf{H}^{s(l)}\left(\mathbf{r}_{\mathrm{R}_{1}}\right), \delta \mathbf{H}^{s(l)}\left(\mathbf{r}_{\mathrm{R}_{2}}\right), \ldots, \delta \mathbf{H}^{s(l)}\left(\mathbf{r}_{\mathrm{R}_{\mathrm{M}}}\right)\right]^{t},
$$

in which $M$ is the number of individual measurements. The vector a consists of the conductivity corrections,

$$
\mathbf{a}=\left[\delta \sigma_{1}^{(l+1)}, \delta \sigma_{2}^{(l+1)}, \ldots, \delta \sigma_{N}^{(l+1)}\right]^{t},
$$

where $N$ is the number of pixels. Here, $\mathbf{K}$ is the sensitivity matrix composed of Fréchet derivatives associated with the individual pixels,

$$
\mathbf{K}=\left(K_{i j}\right)_{M \times N}, \quad K_{i j}=\hat{\alpha}_{R} \cdot \int_{V_{j}} d \mathbf{r}^{\prime} \nabla \times \overline{\mathbf{G}}_{e}^{(l)}\left(\mathbf{r}_{\mathrm{R}_{\mathrm{i}}}, \mathbf{r}^{\prime}\right) \cdot \mathbf{E}^{(l)}\left(\mathbf{r}^{\prime}, \mathbf{r}_{\mathrm{T}_{i}}\right) .
$$

The ill-posedness of the inverse scattering problem inhibits a direct solution of (3.36). Hence, one considers the following optimization problem:

$$
\Phi(\mathbf{a})=(\mathbf{K} \cdot \mathbf{a}-\mathbf{b})^{\dagger} \cdot \mathbf{W}_{d} \cdot(\mathbf{K} \cdot \mathbf{a}-\mathbf{b})+\gamma \mathbf{a}^{\dagger} \cdot \mathbf{W}_{m} \cdot \mathbf{a},
$$

where $\gamma$ is the regularization parameter and the superscript $\dagger$ denotes the conjugate transpose. The weighting matrix $\mathbf{W}_{d}$ applies a larger weight to the data which are more sensitive to the unknown parameters. Thus normalized, the data becomes much less dependant on the frequency and on the 
distance from the inversion domain. The other matrix, $\mathbf{W}_{m}$, regularizes the ill-conditioned system. The weighting matrices are defined as [23]

$$
\begin{aligned}
& \mathbf{W}_{d}=\operatorname{diag}\left(\sqrt{\sum_{j}\left|K_{i j}\right|^{2}}\right), \\
& \mathbf{W}_{m}=\operatorname{diag}\left(\sqrt{\sum_{i}\left|K_{i j}\right|^{2}}\right) .
\end{aligned}
$$

From (3.40), one obtains

$$
\left(\mathbf{K}^{\dagger} \cdot \mathbf{W}_{d} \cdot \mathbf{K}+\gamma \mathbf{W}_{m}\right) \cdot \mathbf{a}=\mathbf{K}^{\dagger} \cdot \mathbf{W}_{d} \cdot \mathbf{b} .
$$

The solution of (3.43) is sought by the use of the conjugate gradient (CG) method. The CG method is less sensitive to the ill-conditioning of the linear system than a direct solver. Moreover, the matrix-matrix product $\mathbf{K}^{\dagger} \cdot \mathbf{W}_{d} \cdot \mathbf{K}$ is avoided by CG. When the size of Equation (3.36) is small, the singular value decomposition is preferred since the singular value spectrum helps determine the optimal regularization parameter [24].

After $\mathbf{a}$ is solved for, the conductivity profile is updated via

$$
\sigma^{(l+1)}=\sigma^{(l)}+\delta \sigma^{(l+1)} .
$$

If the incremental field $\delta \mathbf{H}^{s(l+1)}$ due to $\sigma^{(l+1)}$ is small enough to within the specified tolerance, the iterations stop. Otherwise, the above linearization is repeated to successively update the conductivity profile until the tolerance is satisfied. The 2-norm

$$
e r r=\frac{\left\|\delta \mathbf{H}^{s(l+1)}-\mathbf{H}^{s}\right\|}{\left\|\mathbf{H}^{s}\right\|}
$$

is used to describe the distance between the simulated data and the real measurement data.

The selection of the regularization parameter follows a practical rule, i.e.,

$$
\gamma_{l}=\gamma_{0} q^{-l}, \quad l=0,1, \ldots, l_{\max } .
$$

Generally $\gamma$ decreases with the progress of iteration. When, however, err does not drop, $\gamma$ will be amplified instead of reduced by the factor of $q$ in the next step. In the inversion of the VETEM data, $\gamma_{0}$ is selected between 1.0 
to 100.0 , while the contraction factor $q$ is selected between 1.25 to 5.0. This heuristic rule is similar to the one used in the Levenberg-Marquart algorithm. In the above, $\mathbf{E}^{(l)}\left(\mathbf{r}^{\prime}, \mathbf{r}_{\mathrm{T}_{i}}\right)$ is the total internal electric field in the conductivity profile $\sigma^{(l)}$ obtained in the last iteration due to the transmitter located at $\mathbf{r}_{\mathrm{T}_{i}}$. Here, the transmitter is approximated by a magnetic dipole with moment $I A$,

$$
\mathbf{E}^{(l)}\left(\mathbf{r}^{\prime}, \mathbf{r}_{\mathrm{T}_{i}}\right)=-i \omega \mu_{0} I A \nabla \times \overline{\mathbf{G}}_{m}^{(l)}\left(\mathbf{r}^{\prime}, \mathbf{r}_{\mathrm{T}_{i}}\right) \cdot \hat{\alpha}_{T}
$$

where $\hat{\alpha}_{T}$ is the polarization of the transmitter. If $\sigma^{(l)}=\sigma^{(0)}$ or $\sigma_{b}, \overline{\mathbf{G}}_{m}^{(l)}\left(\mathbf{r}^{\prime}, \mathbf{r}_{\mathrm{T}_{i}}\right)$ is computed by the evaluation of Sommerfeld integrals. Generally, when the background medium is inhomogeneous, it is computed numerically by the BCGS-FFT method.

Direct computation of $\nabla \times \overline{\mathbf{G}}_{e}^{(l)}\left(\mathbf{r}_{\mathrm{R}_{\mathrm{i}}}, \mathbf{r}^{\prime}\right)$ is prohibitively time-consuming; hence, we resort to the reciprocity theorem to lower the computational load. In inhomogeneous media [13]

$$
\mathbf{H}(\mathbf{r})=\int d \mathbf{r}^{\prime} \mu^{-1}(\mathbf{r}) \nabla \times \overline{\mathbf{G}}_{e}\left(\mathbf{r}, \mathbf{r}^{\prime}\right) \mu\left(\mathbf{r}^{\prime}\right) \cdot \mathbf{J}\left(\mathbf{r}^{\prime}\right) .
$$

By duality, for the magnetic current source

$$
\mathbf{E}(\mathbf{r})=-\int d \mathbf{r}^{\prime} \varepsilon^{-1}(\mathbf{r}) \nabla \times \overline{\mathbf{G}}_{m}\left(\mathbf{r}, \mathbf{r}^{\prime}\right) \varepsilon\left(\mathbf{r}^{\prime}\right) \cdot \mathbf{M}\left(\mathbf{r}^{\prime}\right) .
$$

We consider nonmagnetic media, so $\mu^{-1}$ and $\mu$ cancel each other in (3.48). Here, the electric current $\mathbf{J}_{1}(\mathbf{r})$ is inside $V_{1}$, which is in the earth, whereas the magnetic current $\mathbf{M}_{2}(\mathbf{r})$ is enclosed in $V_{2}$ above the ground. The magnetic field excited by $\mathbf{J}_{1}(\mathbf{r})$ is

$$
\mathbf{H}_{1}(\mathbf{r})=\int_{V_{1}} d \mathbf{r}^{\prime} \nabla \times \overline{\mathbf{G}}_{e}\left(\mathbf{r}, \mathbf{r}^{\prime}\right) \cdot \mathbf{J}_{1}\left(\mathbf{r}^{\prime}\right)
$$

where the observation point is assumed to be in the earth. Likewise, the electric field excited by $\mathbf{M}_{2}(\mathbf{r})$ is

$$
\mathbf{E}_{2}(\mathbf{r})=-\frac{\varepsilon_{a}}{\varepsilon_{b}} \int_{V_{2}} d \mathbf{r}^{\prime} \nabla \times \overline{\mathbf{G}}_{m}\left(\mathbf{r}, \mathbf{r}^{\prime}\right) \cdot \mathbf{M}_{2}\left(\mathbf{r}^{\prime}\right),
$$

where it is assumed that the observation point is in the air. Here, $\varepsilon_{a}$ and $\varepsilon_{b}$ are relative permittivities of the air and the earth, respectively. It follows from the reciprocity theorem that $\left\langle\mathbf{M}_{2}, \mathbf{H}_{1}\right\rangle=-\left\langle\mathbf{J}_{1}, \mathbf{E}_{2}\right\rangle$. Then, 


$$
\begin{gathered}
\int_{V_{2}} \int_{V_{1}} d \mathbf{r} d \mathbf{r}^{\prime} \mathbf{M}_{2}(\mathbf{r}) \cdot \nabla \times \overline{\mathbf{G}}_{e}\left(\mathbf{r}, \mathbf{r}^{\prime}\right) \cdot \mathbf{J}_{1}\left(\mathbf{r}^{\prime}\right)= \\
\frac{\varepsilon_{a}}{\varepsilon_{b}} \int_{V_{2}} \int_{V_{1}} d \mathbf{r} d \mathbf{r}^{\prime} \mathbf{J}_{1}\left(\mathbf{r}^{\prime}\right) \cdot \nabla^{\prime} \times \overline{\mathbf{G}}_{m}\left(\mathbf{r}^{\prime}, \mathbf{r}\right) \cdot \mathbf{M}_{2}(\mathbf{r})
\end{gathered}
$$

Hence,

$$
\nabla \times \overline{\mathbf{G}}_{e}\left(\mathbf{r}, \mathbf{r}^{\prime}\right)=\frac{\varepsilon_{a}}{\varepsilon_{b}}\left[\nabla^{\prime} \times \overline{\mathbf{G}}_{m}\left(\mathbf{r}^{\prime}, \mathbf{r}\right)\right]^{t}
$$

Substituting (3.47) and (3.53) into (3.39), we have

$$
K_{i j}=-i \omega \mu_{0} I A \frac{\varepsilon_{a}}{\varepsilon_{b}} \hat{\alpha}_{R} \cdot \int_{V_{j}} d \mathbf{r}^{\prime}\left[\nabla^{\prime} \times \overline{\mathbf{G}}_{m}^{(l)}\left(\mathbf{r}^{\prime}, \mathbf{r}_{\mathrm{R}_{\mathrm{i}}}\right)\right]^{t} \cdot \nabla \times \overline{\mathbf{G}}_{m}^{(l)}\left(\mathbf{r}^{\prime}, \mathbf{r}_{\mathrm{T}_{i}}\right) \cdot \hat{\alpha}_{T}
$$

Equation (3.54) indicates that for each data point, only two calls to the forward solver are needed to calculate the derivatives of the data with respect to all the pixels. The first calculates the internal electric field generated by a magnetic point source with moment $I A$ at the transmitter, whereas the second calculates the internal electric field generated by a unit magnetic point source at the receiver. Repeating this process for all data points and all frequencies, the sensitivity matrix $\mathbf{K}$ is filled efficiently.

\subsubsection{Localized 3D Inversion}

A naive implementation of the localized inversion may lead to undesired results when there is strong coupling from the surrounding domains. As shown in Figure 3.18, when $V_{1}$ and $V_{2}$ are close to the subdomain $V$, the response is distorted, especially around the edges of $V$. However, when the inversion is performed over $V$, the effects of $V_{1}$ and $V_{2}$ are not considered by the algorithm. Thus, the inversion algorithm is misled by the data associated with $V$ and false images are produced. This effect is appropriately named as an "edge effect."

Two practical methods are used to suppress the edge effect. The first is to use weighting for measurements. One possible choice is $\cos ^{2}\left(\frac{x}{2}\right)$, where $x$ is the normalized distance from the subdomain center. If the radius of the smallest sphere encompassing all the data points associated with the subdomain $V$ is $a$ with center at $\left(x_{c}, y_{c}\right)$, then for any data at point $(x, y)$, the normalized distance is $\frac{\sqrt{\left(x-x_{c}\right)^{2}+\left(y-y_{c}\right)^{2}}}{a}$. 

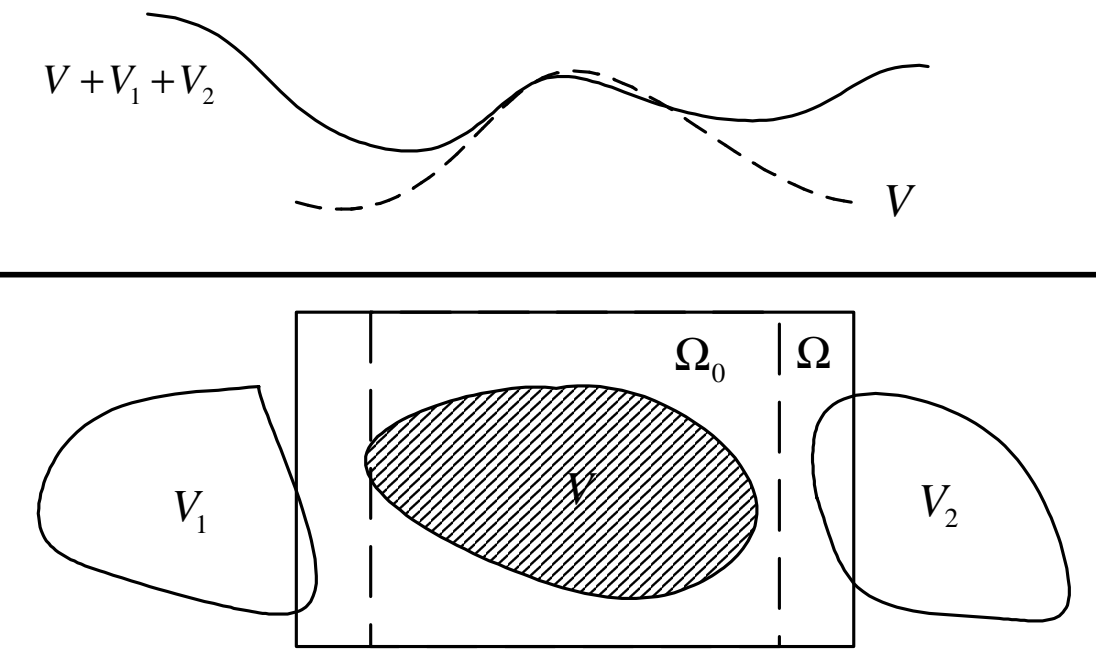

Figure 3.18: The effect of surrounding objects on the local inversion area.

The second method involves the use of a larger inversion area, as shown in Figure 3.18. The new area $\Omega$ encompasses the original $\Omega_{0}$ in the radial direction. The added size in each direction should not be less than the characteristic distance of the VETEM tool, i.e., the 2-m distance between the transmitter and the receiver.

\subsubsection{Validation of 3D Inversion with Synthetic Data}

In the following, the notation $\delta$ denotes the skin depth, equal to the reciprocal of the imaginary part of wave number, or $1.0 / \operatorname{Im}(k)$. Mean skin depth is defined as $\delta_{m}=\sum_{k=1}^{K} \delta_{k} / K$, which is the arithmetic average of the skin depths related to the frequencies used in an inversion process. The notation $d$ is the estimated burial depth, which is the depth of the bottom of buried objects, defining the lower boundary of the area to be reconstructed. The burial depth is chosen according to the mean skin depth calculated with the knowledge of frequencies and the background conductivity. The height of the VETEM system $h$ is $0.53 \mathrm{~m}$ above the ground surface.

First the inversion code is tested on synthetic data. The original model is shown in Figure 3.19. There are two conductive boxes buried in the earth, the sizes of which are both $2 \mathrm{~m} \times 3 \mathrm{~m} \times 1 \mathrm{~m}$. The burial depths are $3 \mathrm{~m}$ and $2.5 \mathrm{~m}$ respectively. The shallower one has a conductivity of $0.05 \mathrm{~S} / \mathrm{m}$ and the deeper one $0.1 \mathrm{~S} / \mathrm{m}$. The earth conductivity is $0.01 \mathrm{~S} / \mathrm{m}$. The permittivity 

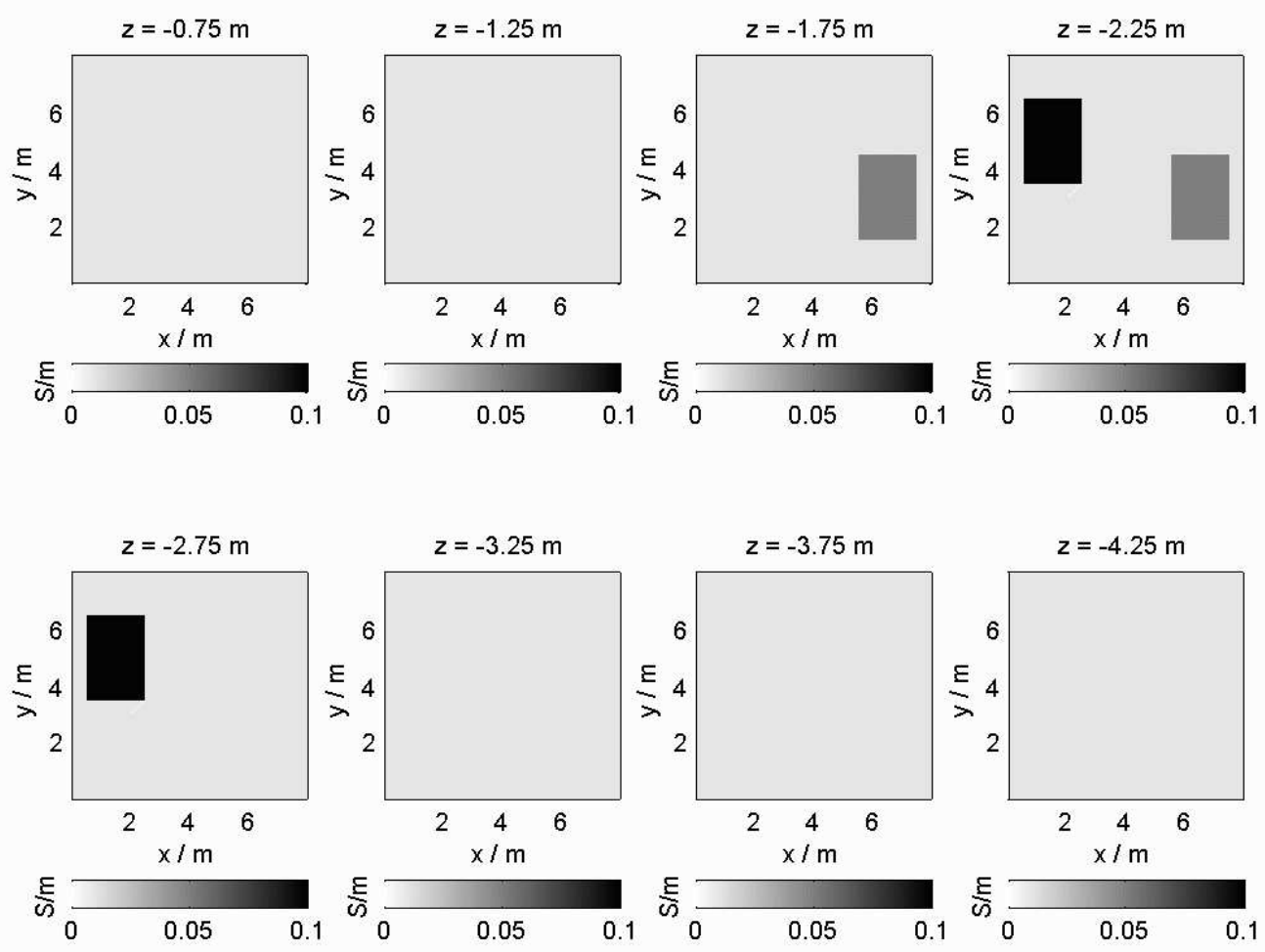

Figure 3.19: The true model used to generate the synthetic data. Two conductive objects of the same size are embedded in the resistive earth; the deeper one is more conductive than the shallower one.

remains constant everywhere and is set to 16 . The background conductivity and the permittivity are assumed known in the inversion. The six frequencies ranging from $0.062 \sim 3.052 \mathrm{MHz}$ with the sampling step of $0.299 \mathrm{MHz}$ are in line with those used in the field data processing, as mentioned in the introduction.

The synthetic data is generated using the forward solver BCGS-FFT, and then is read into the DBIM code as input. The mean skin depth $\delta_{m}$ for this case is $7.0 \mathrm{~m}$. However, we assume the burial depth is $4.5 \mathrm{~m}$ for simplicity, which is larger than the actual value. The area to be inverted is $8 \mathrm{~m} \times 8 \mathrm{~m}$ $\times 4 \mathrm{~m}$, the bottom of which is at the chosen burial depth. It is separated 
into $16 \times 16 \times 8$ pixels, the conductivities of which are to be solved for in the inversion. The data are sampled in a horizontal plane with size 8 $\mathrm{m} \times 8 \mathrm{~m}$ below which is located the inversion area, and totally $6 \times 8 \times 8$ synthetic data are obtained. The position of data point $\left(x_{i}, y_{j}\right)$ is obtained by $x_{i}=(i-0.5), y_{j}=(j-0.5), i, j=1, \ldots, 8$.

The number of unknowns of the resulting equation is 2048, while the number of data is 384, and therefore it is greatly unbalanced and underdetermined. The problem is first solved using the noise-free data, and with thirteen iterations, an inverted model is obtained as shown in Figure 3.20. The error err $=0.97 \%$ when the code stops. Here, $\gamma_{0}=4.0 \times 10^{-2}, q=2.5$. It is seen that the magnitude of conductivity is not fully recovered, but the two boxes are clearly resolved, and their relative position is correctly recovered.

The same data set is also processed using the Born approximation (BA) method. The key to the success of BA is the selection of the regularization parameter $\gamma$. Figure 3.21 presents the L-curve which is used for the selection. Note that the misfit is calculated by not only the Born approximation, but also the rigorous solution BCGS-FFT. It is interesting that the optimal $\gamma$ from the two curves are the same. The optimal $\gamma\left(=1.64 \times 10^{-4}\right)$ is then used in BA and the result is shown in Figure 3.22. It is seen that the image become blurred. Though the two objects are still resolved, their burial depth and their relative position are not fully recovered.

The data are then contaminated with $5 \%$ random error and are reprocessed by the DBIM code. It is found that after eight iterations, the code converges to $4.8 \%$ and stops. The history is shown in Figure 3.25. The inverted profile is shown in Figure 3.23. Obviously the image appears to be more vague than the noise-free case, but still better than the BA image even though no noise is added therein. We then add $10 \%$ random error to the original data and this time, the image looks more blurred, especially the vertical resolution, which becomes even worse. However, compared to the BA image, it is still of seemingly better horizontal resolution. The history is also plotted in Figure 3.25. It is seen that the two iteration procedures progress smoothly and descend steadily to until the prescribed stopping criterion is fulfilled. In these two cases, $\gamma_{0}=0.1, q=2.5$.

Theoretically, DBIM has a second order convergence and is faster than the Born iterative method. BA does not consider the multiple scattering inside objects which renders a non-negligible contribution to the measurement for high contrast buried objects, as has been shown previously. Therefore, when it comes to processing the data from susceptible high-contrast objects, it is 

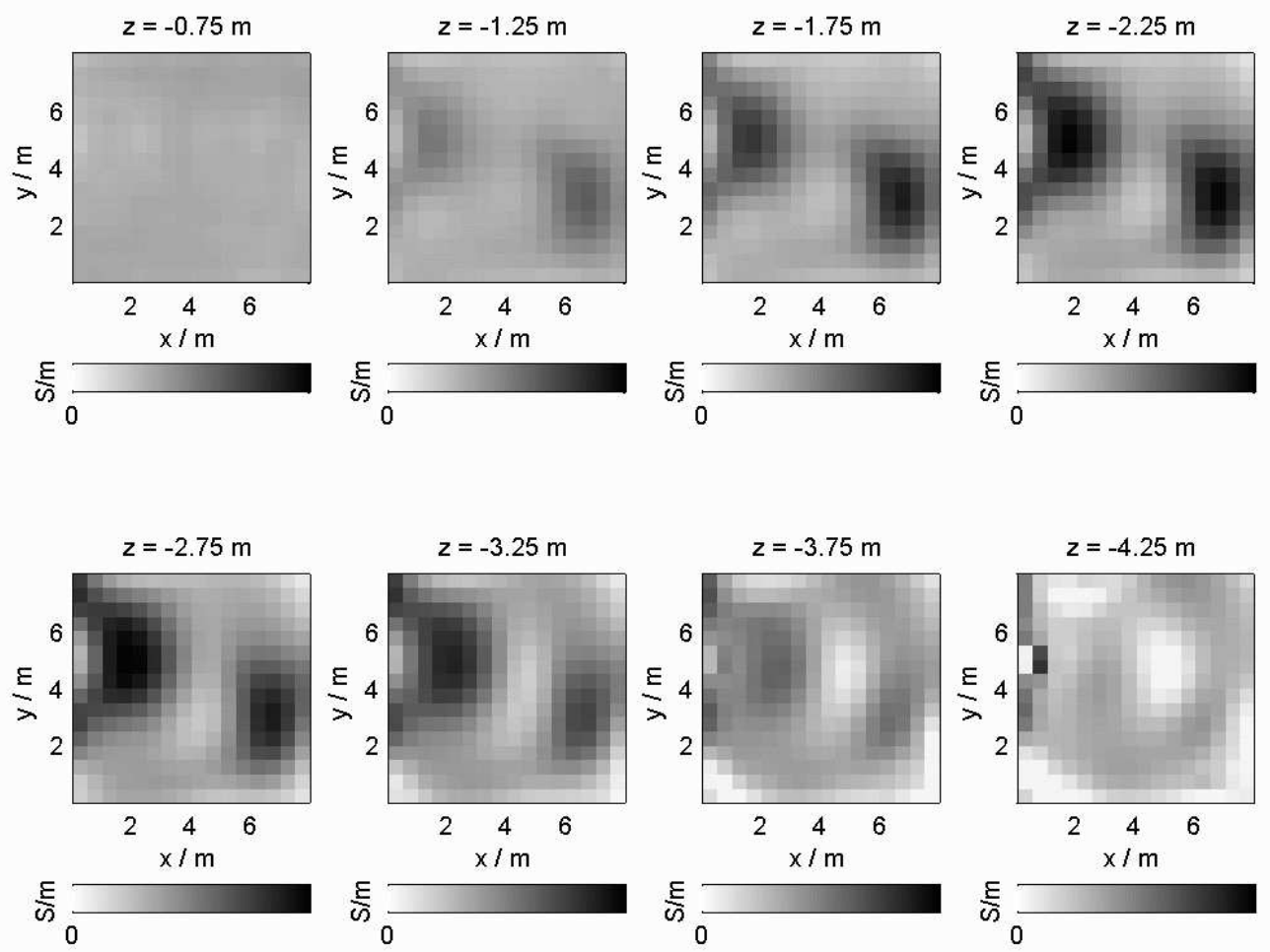

Figure 3.20: The model reconstructed with the distorted Born iterative method using the data without contamination. Here, $\gamma_{0}=4.0 \times 10^{-2}, q=2.5$. 


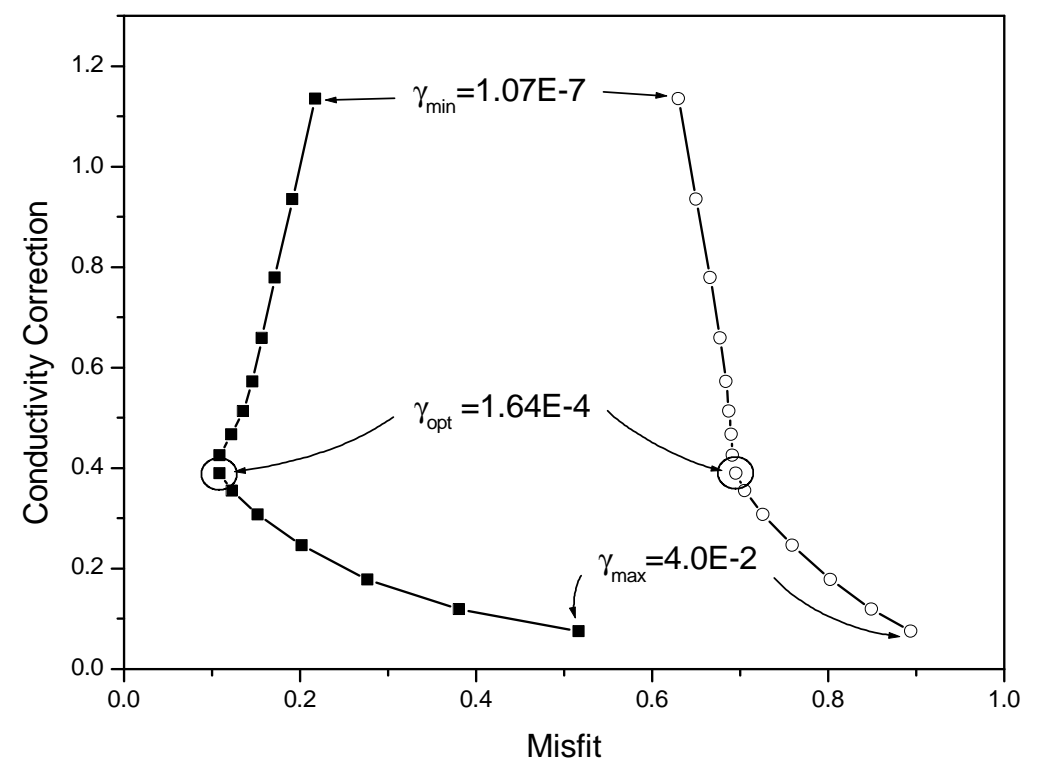

Figure 3.21: L-curve generated by the Born approximation and the rigorous solution BCGS-FFT. The left curve with solid squares is generated by the BCGSFFT method, and the right curve with hollow circles is generated by the Born approximation. The point corresponding to the optimal regularization parameter is enclosed by a large hollow circle. 

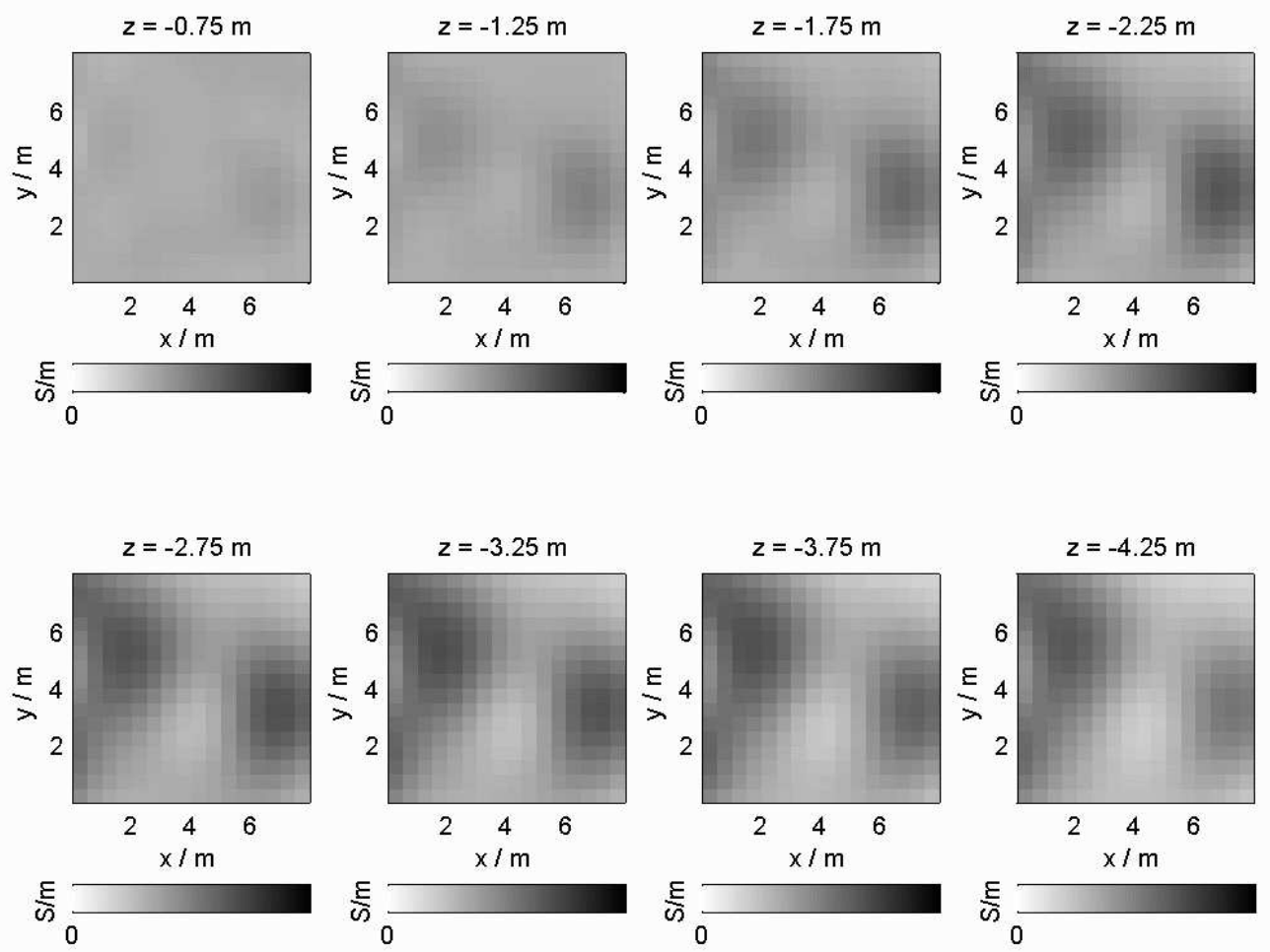

Figure 3.22: The model reconstructed with the Born approximation method using the data without contamination. Here, $\gamma=0.164 \times 10^{-4}$. 

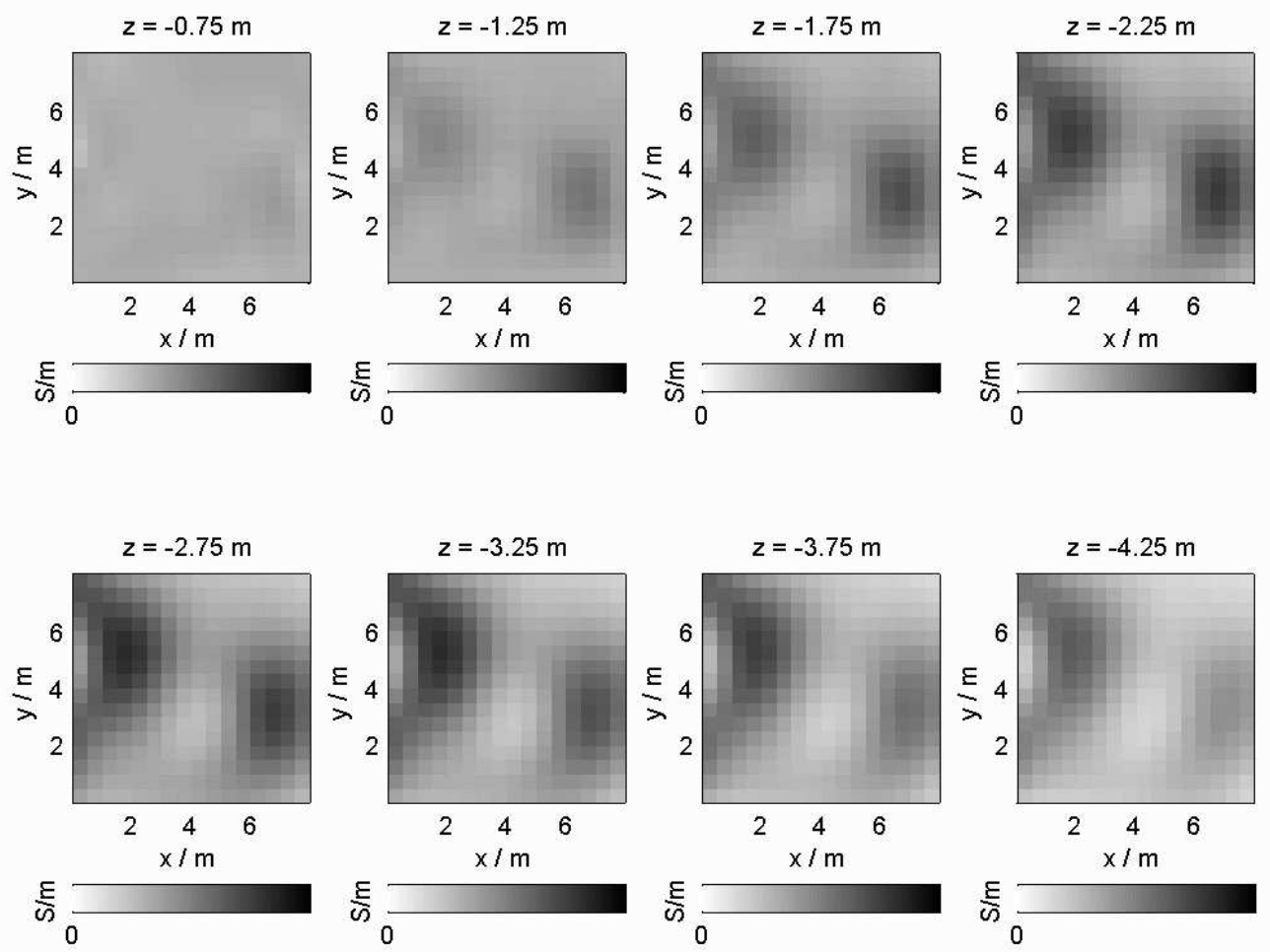

Figure 3.23: The model reconstructed with the distorted Born iterative method using the data with $5 \%$ random error added. Here, $\gamma_{0}=4.0 \times 10^{-2}, q=2.5$. 

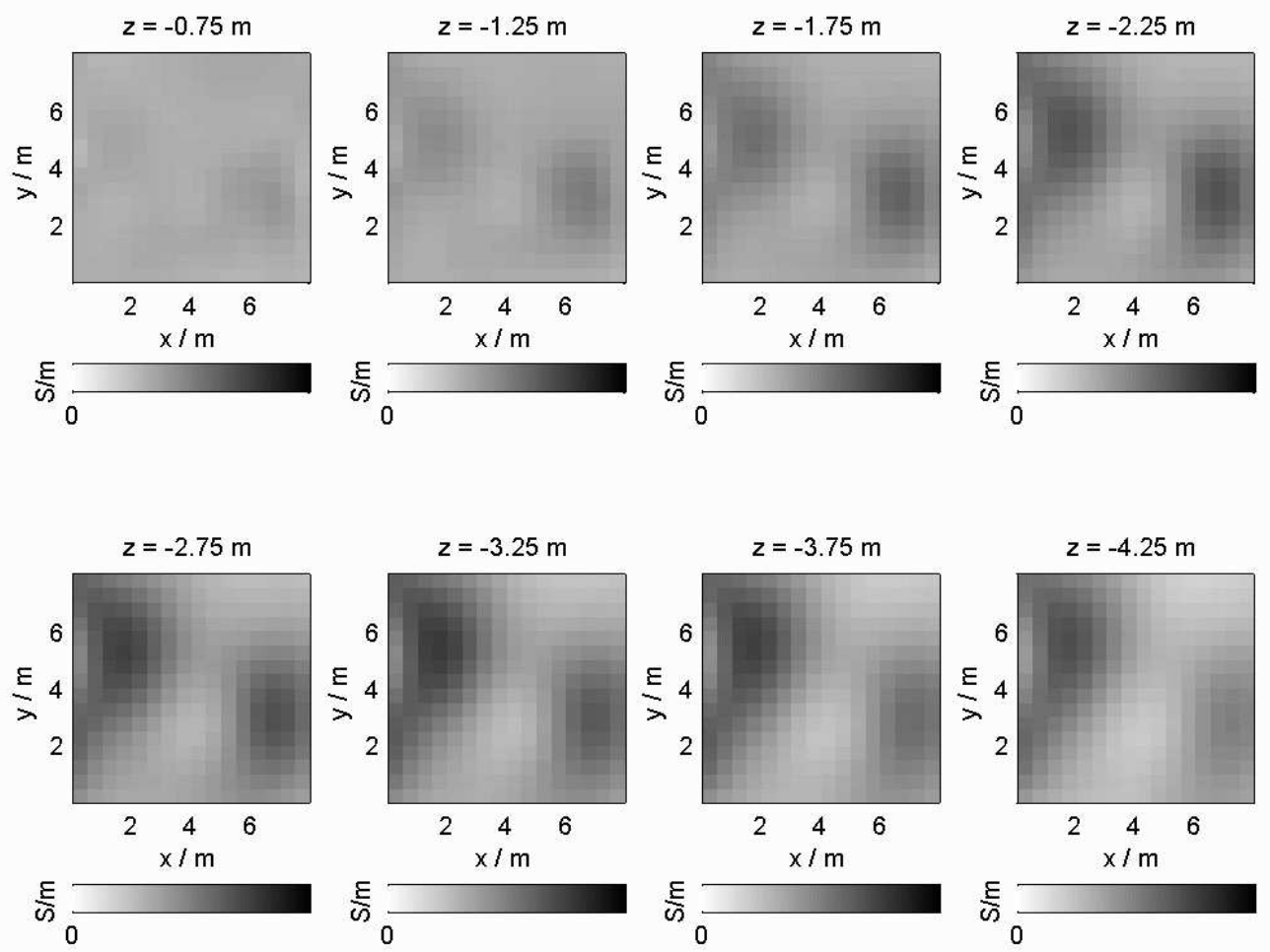

Figure 3.24: The model reconstructed with the distorted Born iterative method using the data with $10 \%$ random error added. Here, $\gamma_{0}=4.0 \times 10^{-2}, q=2.5$. 


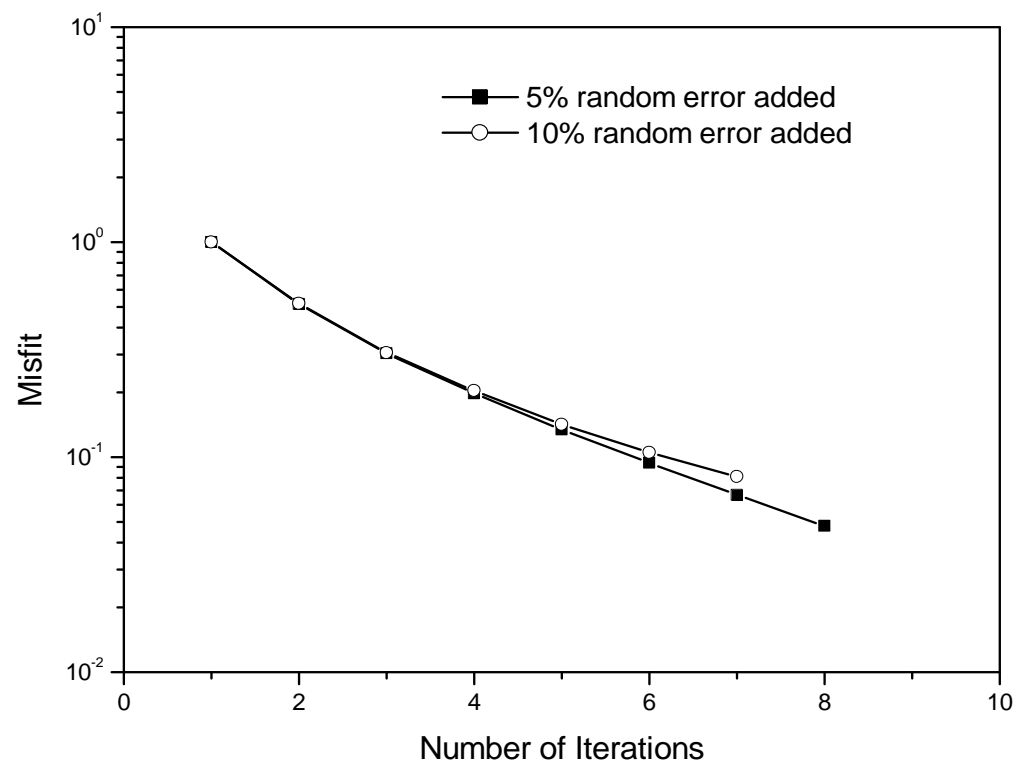

Figure 3.25: The iteration histories for the DBIM processes in Figure 3.23 and Figure 3.24.

better that DBIM is used to achieve a high-resolution subsurface image. For the noisy data processing, the DBIM code generally reconstructs the model fairly well even when the data are contaminated by the random error up to $10 \%$. The relative position and the relative conductivity are recovered in the image. The horizontal resolution appears to be better than the vertical resolution. Increasing the spanning and sampling of the data in the horizontal direction and the sampling in frequency is expected to improve the reconstructed image. However this may increase the computational overhead substantially, and therefore is avoided in this research.

\subsubsection{Application of 3D Inversion to Field Data}

DOE Pit 9 complex is one of many DOE facilities where man-made wastes are buried underneath. The electromagnetic measurement using the VETEM system has been performed at the Pit 9 complex to survey the burial situation of the underground wastes. The original data are in time domain. Fourier transform is used to convert the data to frequency domain [6, 24]. Six frequencies are selected, ranging from $0.062 \mathrm{MHz}$ to $3.052 \mathrm{MHz}$ with the sampling step of $0.299 \mathrm{MHz}$, from the broad frequency spectrum. The principle for 
the selection is that the number of selected frequencies is made small while preserving the information as much as possible.

The conductivity $\sigma_{b}$ and the relative permittivity $\varepsilon_{r b}$ of the earth are $0.03 \mathrm{~S} / \mathrm{m}$ and 16 respectively, which are estimated from the high frequency component of the measurement data that is assumed to contain negligible contribution from buried objects. Readers are referred to $[6,24]$ for details. The mean skin depth $\delta_{m}$ for such background media is $4.0 \mathrm{~m}$. As a result, such burial depth as $5.0 \mathrm{~m}$ is assumed which ensures that the signal coming below this depth has no appreciable contribution to the measurement data.

The measurement area is $72 \mathrm{~m} \times 25.315 \mathrm{~m}$. The magnitude of the data corresponding to the six frequencies are plotted in Figure 3.26. Different scales are used for different frequencies to present the details. Notice that the data at $0.062 \mathrm{MHz}$ does not correlate well with the data at other frequencies. Also notice that at the middle, lower and slightly right part $([25 \mathrm{~m}, 55 \mathrm{~m}] \times[0 \mathrm{~m}$, $8 \mathrm{~m}]$ ), the data consistency is seemingly poor. There are many factors which may affect the data quality, such as the measurement cart wobbling over the unlevel ground surface, the tilt of the transmitter-receiver, the inconsistent calibration factors for different regions, and the environmental electromagnetic noise, to mention a few. These factors invariably worsen the data.

For example, the inconsistent calibration factor undesirably renders the same data look different, and consequently leads to some apparently artificial line features as is shown in the data. And the tilt of the transmitter-receiver results in a strong effect especially on the low frequency data. When the frequency becomes lower, the direct coupling between the transmitter and the receiver, even if their tilt angle is pretty small, becomes increasingly larger. Therefore it easily corrupts the useful component of the data. Considering that it is the scattered field data that are used, the situation becomes even worse because the erroneous signal accounts for a larger part than in the total field data. However quantitative evaluation of their effects proves difficult which may well be treated in the future.

For the DBIM code to be applicable, the measurement is partitioned into thirty uniform subdomains. The original size of each subdomain is $7.2 \mathrm{~m} \times$ $8.4383 \mathrm{~m} \times 5.0 \mathrm{~m}$ and the partition is $8 \times 8 \times 10$. By using this subdomain data, the reconstruction domain is then augmented by two pixels at each side in the $x$ and $y$ direction respectively, therefore the actual partition is $12 \times$ $12 \times 10$, and the actual size is accordingly increased to $10.8 \mathrm{~m} \times 12.6575 \mathrm{~m}$ $\times 5.0 \mathrm{~m}$. The number of unknowns in the new partition for each subdomain is 1440. For each subdomain and each frequency, there are 60 data points. 

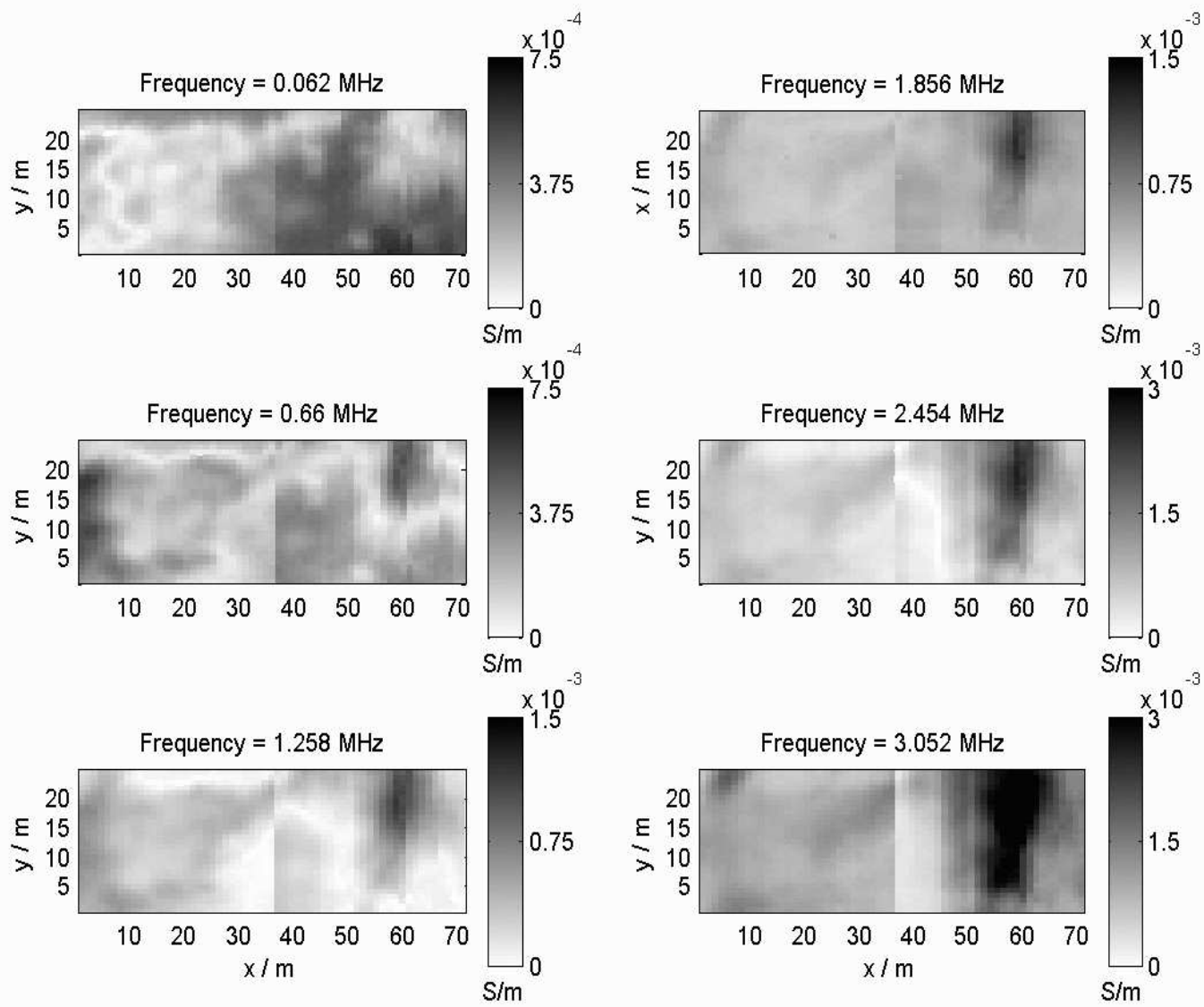

Figure 3.26: The magnitude of the measurement data of the DOE Pit 9 complex for the whole measurement area. 


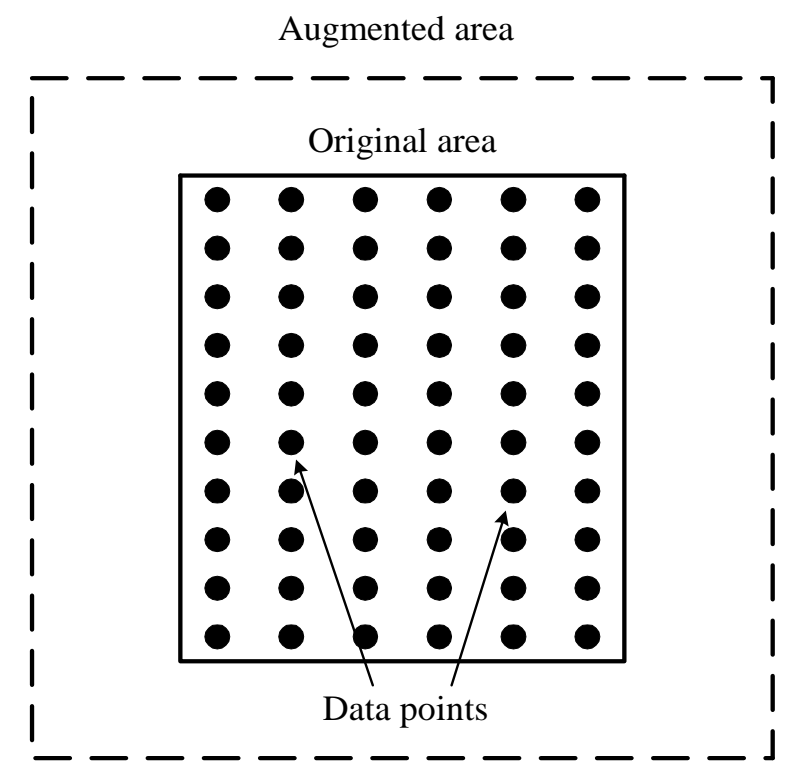

Figure 3.27: The augmented area and the data points used for the conductivity image reconstruction of the DOE Pit 9 complex. The data points are enclosed by the original area.

The original area, the augmented area and the data positions are shown in Figure 3.27. Totally, the number of data is 360, therefore the resulting equation is still underdetermined.

It turns out that the combined image from the images for the thirty subdomains takes on apparent artifacts along the interfaces between adjacent subdomains. We thus introduce the sliding window method (SWM) to suppress the strong effect. SWM is still the localized inversion, but the subdomains overlap each other, the only difference from the original localized inversion. In SWM, the inversion area is likened as a processing window, which moves along either the edge or the diagonal of the subdomains, and therefore forming new subdomains which overlap the original ones. The centers of new subdomains are on the center of edges or vertices of the original subdomains. Figure 3.28 shows four possible patterns of sliding, out of a total of eight being used. By using SWM, there are sixty-five more subdomains to be processed, about two times more than when it is not used.

In the inversion, the initial regularization parameter $\gamma_{0}=0.1$, and the contraction factor $q=2.5$. The maximum iteration count is 10 . It takes about 5-10 mins for each iteration to run in our Pentium 4, 2.4 GHz personal com- 

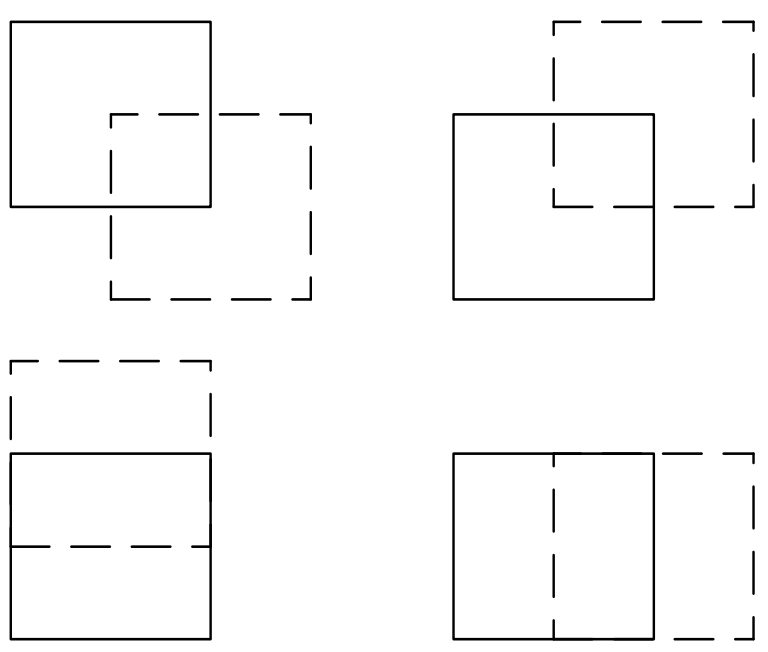

Figure 3.28: Four patterns for sliding of the sliding windows. The boxes with solid line are original sudomains. The boxes with dashed line are new subdomains created by sliding the processing window.

puter, so the time for processing each subdomain is 75 to 90 mins in the same computer.

Combining the images from all subdomains needs interpolation since one point can belong to different subdomains simultaneously. Bi-linear interpolation is used to achieve this goal. The combined image is shown in Figure 3.30. The scattered field data generated synthetically from the reconstructed profile is shown Figure 3.29. Compared to the measurement data in Figure 3.26, it is seen that they are different in terms of the magnitude. However, their patterns are basically consistent, implying that they are correlated well to each other. The relative large discrepancy in the magnitude comes from the stopping criterion used in the inversion. It stipulates that the iteration stops when the iteration number exceeds ten regardless of the difference between the synthetic data and the measurement data. This is sensible considering the apparently large uncertainty and inconsistency in the measurement data. Generally, when the code stops, it is found that err is between $0.4 \sim 0.7$, and for some cases it can be up to 0.9. For example, for the subdomains falling in the middle, lower and slightly right part $([25 \mathrm{~m}, 55 \mathrm{~m}] \times[0 \mathrm{~m}, 8$ $\mathrm{m}]$ ), the err becomes much higher, which reflects the larger inconsistency in the measurement data in this area. The large inconsistency between different frequencies makes it hard for the optimization process to find a solution to 

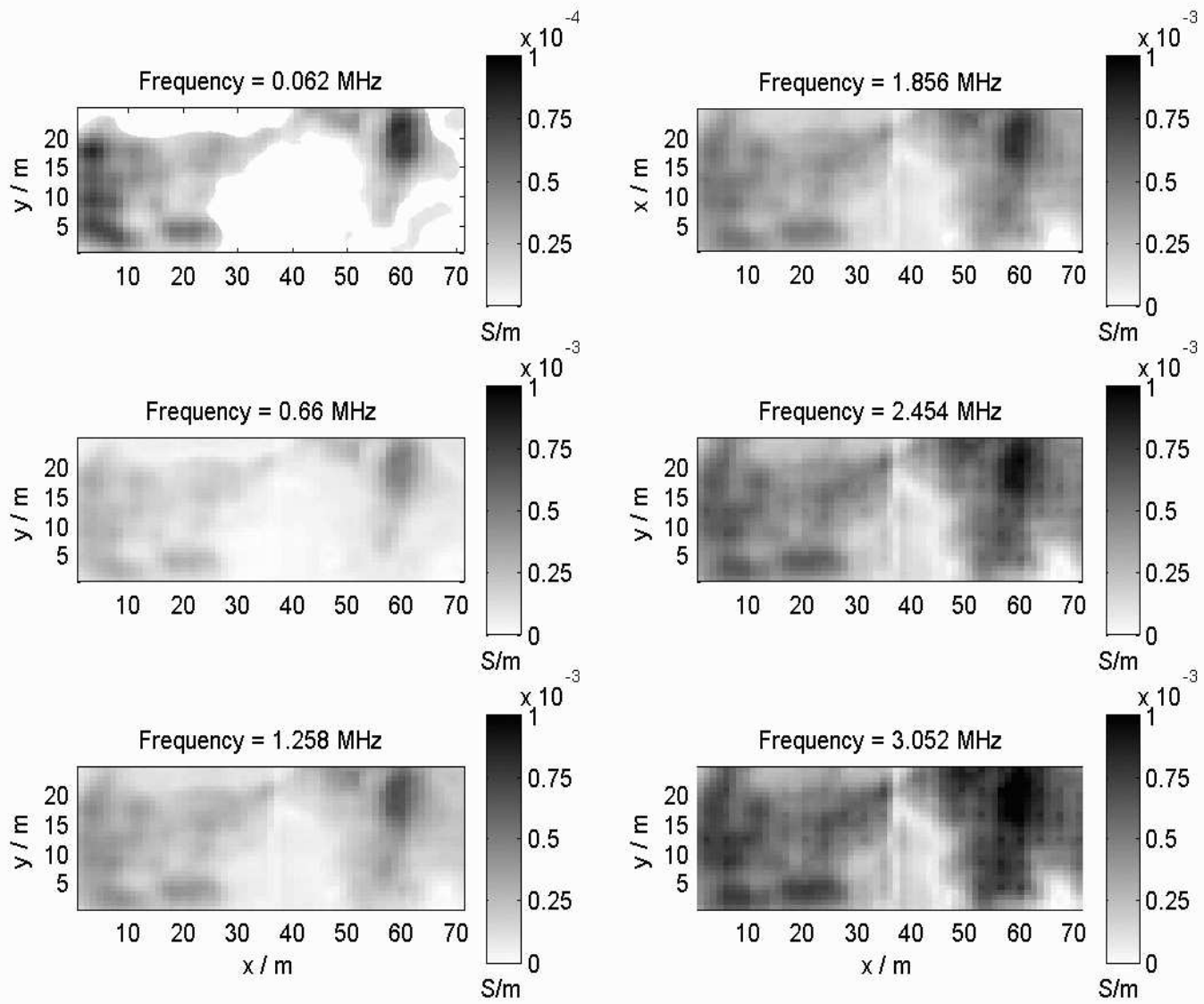

Figure 3.29: The scattered field data generated synthetically from the reconstructed conductivity profile of the DOE Pit 9 complex. 
match all the data.

The same VETEM data set was processed previously using BA, and the result is shown in Figure 3.31. Comparison with the previous result shows that the present image looks better. Not only the artifacts are suppressed substantially, but also the resolution is improved greatly. The image is rich in more details amenable to the analysis of the space variance of the buried objects. From the image, it can be seen that there are high conductive objects buried under the left part of the measurement area, the burial depths of which are between $2 \sim 4 \mathrm{~m}$. Under the right part are the buried objects with even higher conductivity, and their burial depths are between $1 \sim 3 \mathrm{~m}$. Notice that the image at $z=-1.75 \mathrm{~m}$ assumes relative lower conductivity and smaller correlation compared to the adjacent two profiles at $z=-1.25 \mathrm{~m}$ and $z=-2.25 \mathrm{~m}$, which may mean the existence of something interesting at this depth, for example, something which tends to separate the regions above and below this depth.

The two 3D inversion solvers are also applied to process the GEM-2 data collected at DOE Pit 10. Figure 3.32 and Figure 3.33 are respectively DBIM result and the Born inversion result. The depth at the top of each sub-figure is pseudo depth, which is inversely proportional to the square root of the frequency. It is seen that the two profiles are similar to each other, but in the DBIM profile, the conductivity contrast is much higher, meaning that the resolution is higher and the objects are easier to identify. Note that the original data have 17 frequencies and 771 measurement points. For efficiency, only 5 frequencies and 100 points are used for both the Born inversion and the DBIM inversion.

\subsection{Conclusions}

Computational complexity is a crucial element of inverse scattering. However high the quality of a reconstruction scheme may be, computational complexity may make real-world use impossible or dramatically restrict computational area. The 1D inversion scheme scales linearly with computational domain and is therefore promising for large scale geophysical exploration. It is even feasible to apply on-site inversion.

The practical benefits of the 1D method are as follows:

- The simple transmitter, receiver setup performs a single, time domain measurement at each point. 

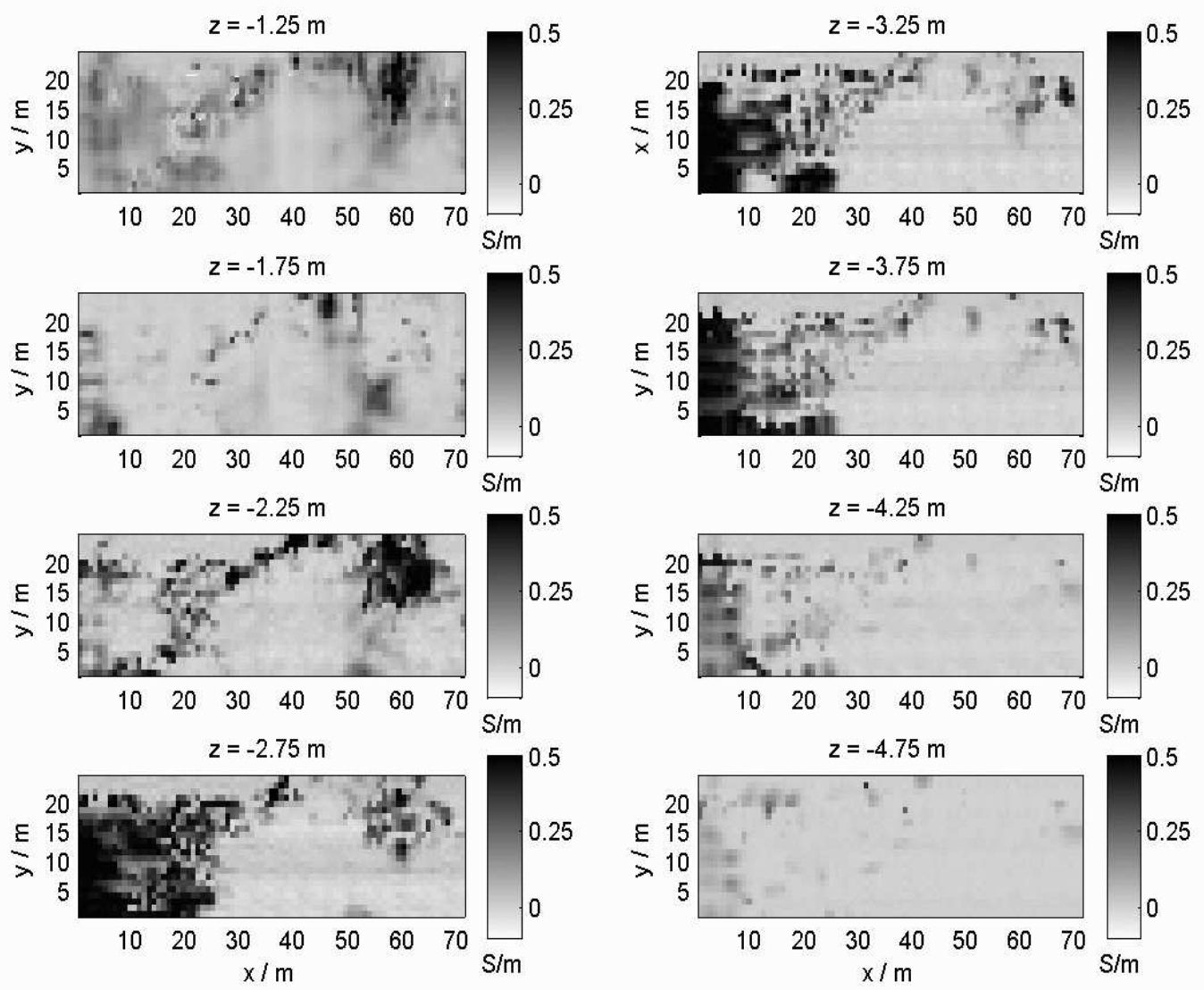

Figure 3.30: The subsurface conductivity image at the DOE Pit9 complex reconstructed using the distorted Born iterative method. 

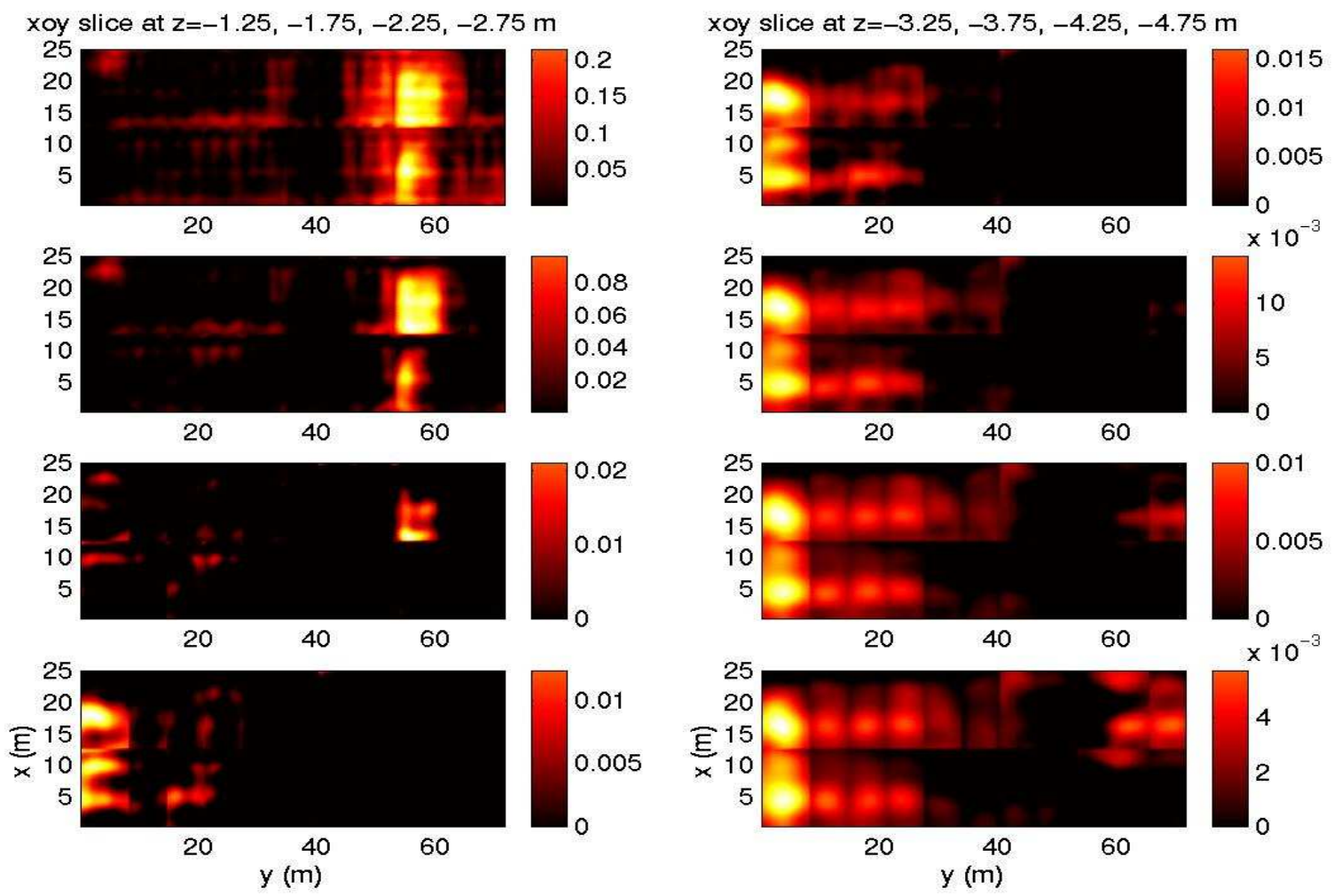

Figure 3.31: The subsurface conductivity image at the DOE Pit9 complex reconstructed by the inversion using the distorted Born iterative method.
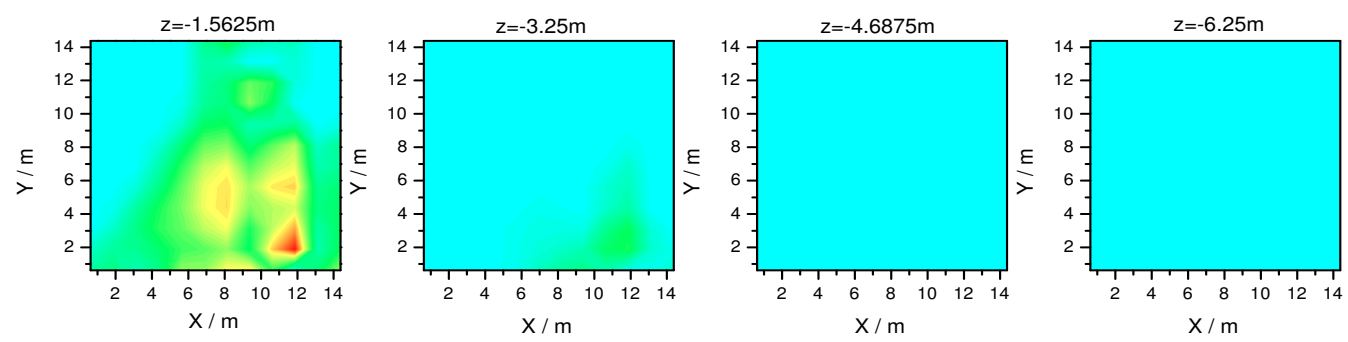

Figure 3.32: The subsurface conductivity image at the DOE Pit9 complex reconstructed by the inversion using the distorted Born iterative method. 

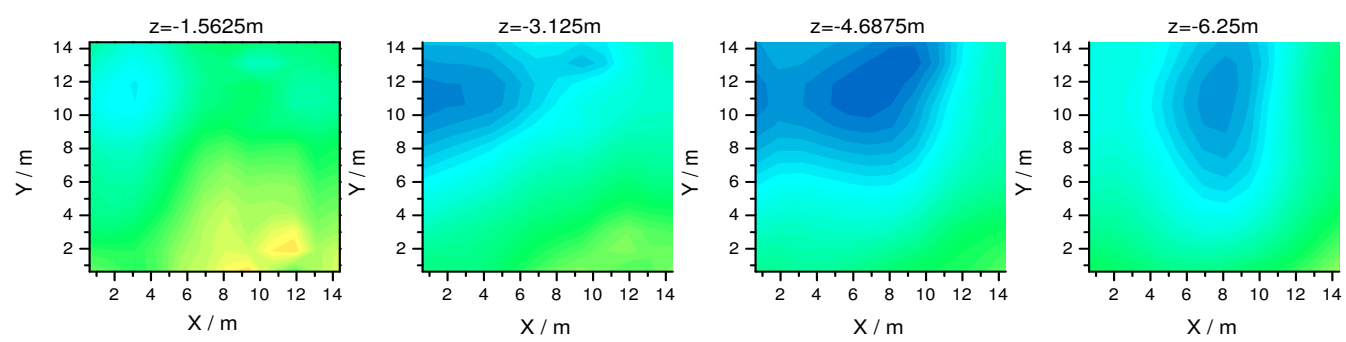

Figure 3.33: The subsurface conductivity image at the DOE Pit9 complex reconstructed by the inversion using the distorted Born iterative method.

- The method can study very large computational domains with $\mathrm{O}(N)$ computational cost.

- Background is estimated at each observation point, thus facilitating the study of inhomogeneous background.

- The method is highly parallelizable by virtue of its $1 \mathrm{D}$ nature and its embarrassing parallelism.

- Preprocessing is computationally less intensive than the inversions themselves and is independent of the type of inversion to follow.

To achieve more reliable subsurface image, two kinds of 3D inverse solver are developed which are used to process the VETEM data and the GEM-2 data. The forward solver is based on the $\mathbf{D}$ formulation BCGS-FFT. The advantage of $\mathbf{D}$ formulation is its continuity along the polarization direction, which gives a better convergence than using $\mathbf{J}$ formulation.

When high conductive objects exist, DBIM is preferred than the Born approximation. The Fréchet derivatives are computed rapidly with the use of the reciprocity theorem, which is otherwise computationally demanding.

The comparison with the Born approximation result shows that the image obtained by DBIM is sharper and clearer. The future direction for the research will include finding more efficient forward algorithm as well as more efficient inverse algorithm for high conductive buried objects. 


\section{Chapter 4}

\section{PUBLICATIONS}

The processing of VETEM data using Born Inversion and DBIM, together with the forward modeling work, have been funded by DOE since 2001. During this period, a number of related papers have been published, listed as follows:

1. G. L. Wang, W. C. Chew, T. J. Cui, A. A. Aydiner, D. L. Wright and D. V. Smith, "3D near-to-surface conductivity reconstruction by inversion of VETEM data using the distorted Born iterative method," Inverse Problem, submitted in April 2004.

2. G. L. Wang and W. C. Chew, "Formal solution to the electromagnetic scattering by a buried sphere," Radio Science, accepted in July 2004.

3. T. J. Cui, A. A. Aydiner, W. C. Chew, D. L. Wright and D. V. Smith, "Three-Dimensional Imaging of Buried Targets in Very Lossy Earth by Inversion of VETEM Data," IEEE Transactions on Geoscience and Remote Sensing, vol. 41, pp. 2197-2210, Oct. 2003.

4. T. J. Cui, W. C. Chew, A. A. Aydiner and Y. H. Zhang, "Fast-forward solvers for the low-frequency detection of buried dielectric objects," IEEE Transactions on Geoscience and Remote Sensing, vol. 41, pp. 2026-2036, Sep. 2003.

5. T. J. Cui, W. C. Chew, A. A. Aydiner, D. L. Wright and D. V. Smith, "3D imaging of buried targets in very lossy earth by inversion of VETEM data," IEEE Trans. on Geoscience and Remote Sensing, submitted in June 2002. 
6. T. J. Cui and W. C. Chew, "Diffraction tomographic algorithm for the detection of three-dimensional objects buried in a lossy half-space," IEEE Transactions on Antennas and Propagation, vol. 50, pp. 42-49, Jan 2002.

7. T. J. Cui, W. C. Chew, A. A. Aydiner, D. L. Wright and D. V. Smith, "Detection of buried targets using a new enhanced very early time electromagnetic (VETEM) prototype system," IEEE Transactions on Geoscience and Remote Sensing, vol. 39, pp. 27022712, Dec. 2001. 


\section{ACKNOWLEDGEMENTS}

This material is based upon work supported by the U.S Department of Energy under award No. DE-FG07-01ID14132. 


\section{Bibliography}

[1] T. J. Cui, W. C. Chew, A. A. Aydiner, D. L. Wright, D. V. Smith, and J. D. Abraham, "Numerical modeling of an enhanced very early time electromagnetic (VETEM) prototype system," IEEE Antennas and Propagation Magazine, vol. 42, pp. 17-27, Apr. 2000. Addendum next issue pp. 54-57.

[2] T. J. Cui, W. C. Chew, A. A. Aydiner, D. L. Wright, and D. V. Smith, "Detection of buried targets using a new enhanced very early time electromagnetic (VETEM) prototype system," IEEE Transactions on Geoscience and Remote Sensing, vol. 39, pp. 2702-2712, Dec. 2001.

[3] F.-C. Chen and W. C. Chew, "An impulse radar nondestructive evaluation system," in Review of Progress in Quantitative Nondestructive Evaluation (D. O. Thompson and D. E. Chimenti, eds.), vol. 16, pp. 709-715, Plenum Press, New York, 1997.

[4] W. H. Weedon and W. C. Chew, "Broadband microwave inverse scattering for nondestructive evaluation (NDE)," in Review of Progress in Quantitative Nondestructive Evaluation (D. O. Thompson and D. E. Chimenti, eds.), vol. 13, pp. 615-622, Plenum Press, New York, 1994.

[5] T. J. Cui and W. C. Chew, "Fast evaluation of Sommerfeld integrals for EM scattering and radiation by three-dimensional buried object," IEEE Transactions on Geoscience and Remote Sensing, vol. 37, pp. 887-900, Mar. 1999.

[6] A. A. Aydiner, W. C. Chew, T. J. Cui, D. L. Wright, D. V. Smith, and J. D. Abraham, "3D imaging of large scale buried structure by $1 \mathrm{D}$ inversion of very early time electromagnetic (VETEM) data," IEEE 
Transactions on Geoscience and Remote Sensing, vol. 39, pp. 1307-1315, June 2001.

[7] A. A. Aydiner, W. C. Chew, T. J. Cui, D. L. Wright, D. V. Smith, and J. D. Abraham, "A 1D inversion scheme for large-scale, 3D subsurface imaging of real data," in IEEE Antennas and Propagation International Symposium, vol. 1, pp. 284-287, June 2002.

[8] P. Chaturverdi and R. G. Plumb, "Electromagnetic imaging of underground targets using constrained optimization," IEEE Transactions on Geoscience and Remote Sensing, vol. 33, pp. 551-561, May 1995.

[9] N. Zaiping and Z. Yerong, "Hybrid Born iterative method in lowfrequency inverse scattering problem," IEEE Transactions on Geoscience and Remote Sensing, vol. 36, pp. 749-753, May 1998.

[10] N. Joachimowicz, C. Pichot, and J. P. Hugonin, "Inverse scattering: An iterative numerical method for electromagnetic imaging," IEEE Transactions on Antennas and Propagation, vol. 39, pp. 1742-1752, Dec. 1991.

[11] H. T. Lin and Y. W. Kiang, "Microwave imaging for a dielectric cylinder," IEEE Transactions on Microwave Theory and Techniques, vol. 42, pp. 1572-1579, Aug. 1994.

[12] W. C. Chew and Y. M. Wang, "Reconstruction of two-dimensional permittivity distribution using the distorted Born iterative method," IEEE Transactions on Medical Imaging, vol. 9, pp. 218-225, June 1990.

[13] W. C. Chew, Waves and Fields in Inhomogeneous Media. New York: IEEE Press, 1995.

[14] Y. M. Wang and W. C. Chew, "Limited-angle inverse scattering problems and their applications for geophysical explorations," International Journal of Imaging Systems and Technology, vol. 2, pp. 96-111, Summer 1990.

[15] W. C. Chew and Q.-H. Liu, "Inversion of induction tool measurements using the distorted Born iterative method and CGFFHT," IEEE Transactions on Geoscience and Remote Sensing, vol. 32, pp. 878-884, July 1994. 
[16] T. M. Habeshy, E. Y. Chow, and D. G. Dudley, "Profile inversion using the renormalized source-type integral equation approach," IEEE Transactions on Antennas and Propagation, vol. 38, pp. 668-682, May 1990.

[17] D. L. Wright, D. V. Smith, J. D. Abraham, R. S. Hutton, T. J. Cui, A. A. Aydiner, and W. C. Chew, "Imaging and modeling new VETEM data," in Proceedings of the Eighth International Conference on GPR, (Australia), pp. 146-150, May 2000.

[18] G. L. Wang, W. C. Chew, T. J. Cui, A. A. Aydiner, D. L. Wright, and D. V. Smith, "3D near-to-surface conductivity reconstruction by inversion of VETEM data using the distorted Born iterative method," submitted to Inverse Problems in early 2004.

[19] P. Zwamborn and van den Berg, "The three-dimensional weak form of the conjugate gradient FFT method for solving scattering problems," IEEE Transactions on Microwave Theory and Techniques, vol. 40, pp. 1757-1766, Sept. 1992.

[20] T. J. Cui and W. C. Chew, "Fast algorithm for electromagnetic scattering by buried 3-D dielectric objects of large size," IEEE Transactions on Geoscience and Remote Sensing, vol. 37, pp. 2597-2608, Sept. 1999.

[21] X. Millard and Q. H. Liu, "A fast volume integral equation solver for electromagnetic scattering from large inhomogeneous objects in planarly layered media," IEEE Transactions on Antennas and Propagation, vol. 51, pp. 2393-2401, Sept. 2003.

[22] R. Barrett, M. Berry, T. F. Chan, J. Demmel, J. Donato, J. Dongarra, V. Eijkhout, R. Pozo, C. Romine, and H. V. der Vorst, Templates for the Solution of Linear Systems: Building Blocks for Iterative Methods. Philadelphia: Society for Industrial and Applied Mathematics, 1994.

[23] M. Zhdanov and G. Hursan, "3D electromagnetic inversion based on quasi-analytical approximation," Inverse Problems, vol. 16, pp. 12971322, 2000.

[24] T. J. Cui, A. A. Aydiner, W. C. Chew, D. L. Wright, and D. V. Smith, "Three-dimensional imaging of buried objects in very lossy earth by inversion of VETEM data," IEEE Transactions on Geoscience and Remote Sensing, vol. 41, pp. 2197-2210, Oct. 2003. 